\title{
Transcription factor NFAT5 promotes macrophage survival in rheumatoid arthritis
}

\author{
Susanna Choi, ${ }^{1}$ Sungyong You, ${ }^{2}$ Donghyun Kim, ${ }^{1}$ Soo Youn Choi, ${ }^{3}$ H. Moo Kwon, ${ }^{3}$ Hyun-Sook Kim, ${ }^{4}$ Daehee Hwang, ${ }^{5}$ \\ Yune-Jung Park, ${ }^{1,6}$ Chul-Soo Cho, ${ }^{1,6}$ and Wan-Uk Kim ${ }^{1,6}$ \\ 'Center for Integrative Rheumatoid Transcriptomics and Dynamics, The Catholic University of Korea, Seoul, Republic of Korea. 2Department of Surgery and Biomedical Sciences, Division of Urology, \\ Cedars-Sinai Medical Center, Los Angeles, California, USA. ${ }^{3}$ School of Nano-Bioscience and Chemical Engineering, Ulsan National Institute of Science and Technology, Ulsan, Republic of Korea. \\ ${ }^{4}$ Department of Internal Medicine, Soonchunhyang University College of Medicine, Seoul, Republic of Korea. ${ }^{5}$ Center for Systems Biology of Plant Senescence and Life History, Institute for Basic Science, \\ Daegu Gyeongbuk Institute of Science and Technology, Daegu, Republic of Korea. ${ }^{6}$ Department of Internal Medicine, College of Medicine, The Catholic University of Korea, Seoul, Republic of Korea.
}

\begin{abstract}
Defective apoptotic death of activated macrophages has been implicated in the pathogenesis of rheumatoid arthritis (RA). However, the molecular signatures defining apoptotic resistance of RA macrophages are not fully understood. Here, global transcriptome profiling of RA macrophages revealed that the osmoprotective transcription factor nuclear factor of activated T cells 5 (NFAT5) critically regulates diverse pathologic processes in synovial macrophages including the cell cycle, apoptosis, and proliferation. Transcriptomic analysis of NFAT5-deficient macrophages revealed the molecular networks defining cell survival and proliferation. Proinflammatory M1-polarizing stimuli and hypoxic conditions were responsible for enhanced NFAT5 expression in RA macrophages. An in vitro functional study demonstrated that NFAT5-deficient macrophages were more susceptible to apoptotic death. Specifically, CCL2 secretion in an NFAT5-dependent fashion bestowed apoptotic resistance to RA macrophages in vitro. Injection of recombinant CCL2 into one of the affected joints of $\mathrm{Nfat5}^{+/-}$mice increased joint destruction and macrophage infiltration, demonstrating the essential role of the NFAT5/CCL2 axis in arthritis progression in vivo. Moreover, after intra-articular injection, NFAT5-deficient macrophages were more susceptible to apoptosis and less efficient at promoting joint destruction than were NFAT5-sufficient macrophages. Thus, NFAT5 regulates macrophage survival by inducing CCL2 secretion. Our results provide evidence that NFAT5 expression in macrophages enhances chronic arthritis by conferring apoptotic resistance to activated macrophages.
\end{abstract}

\section{Introduction}

Nuclear factor of activated T cells 5 (NFAT5) is a member of the Rel family of transcription factors (TFs) that shares a conserved DNA-binding domain with NFATc1-4 and NF- $\kappa$ B. Although these TFs all possess a similar DNA-binding sequence (GGAAA), NFAT5 lacks a calcineurin-binding domain and is instead highly sensitive to salt-induced hypertonicity (1). Thus, NFAT5 was originally designated as a tonicity-responsive enhancer-binding protein involved in the protection of cells from hypertonic stress (1). Several recent studies indicate that NFAT5 is not solely regulated by tonicity, but that it can be stimulated in isotonic tissues by various tonicity-independent mechanisms (2-5). We and others have revealed that NFAT5 promotes the expression of a number of proinflammatory genes in macrophages in response to TLR4 activation and also by stimulating the NF- $\kappa \mathrm{B}$ activity $(2,4,5)$. However, the NFAT5 signature involved in diverse functions of macrophages has not yet been systemically analyzed.

Rheumatoid arthritis (RA) is characterized by a massive infiltration of a variety of chronic inflammatory cells, including synovial macrophages, into the synovia, leading to the destruction of

Conflict of interest: The authors have declared that no conflict of interest exists. Submitted: April 5, 2016; Accepted: December 15, 2016.

Reference information: J Clin Invest. 2017;127(3):954-969.

https://doi.org/10.1172/JCl87880. adjacent cartilage and bone (6). Macrophages are one of the most abundant cell types in the RA synovia (6-8), and there is ample evidence that macrophages are activated in RA and can critically drive the progression of RA $(7,8)$. Synovial macrophages are a major source of various cytokines and chemokines, including C-C motif ligand 2 (CCL2), a key chemokine that controls the migration and infiltration of monocytes/macrophages into the inflamed synovium $(6,9)$. The number of macrophages in the RA synovia has a significant correlation with measures of disease activity, including C-reactive protein levels, erythrocyte sedimentation rate, swollen joint count, synovial lining vascularity and thickness, and radiological severity $(10,11)$. Moreover, persistent activation of certain signaling pathways in synovial macrophages, namely NF- $\kappa$ B, STAT3, and PI3K signaling pathways, enhances resistance to apoptosis mediated by Fas/Fas ligand interactions or by cytokine withdrawal, and it can allow macrophages to better survive under the inflammatory conditions of the RA synovium (12-14). As changes in the number of synovial macrophages and the expression of inflammatory products reflect therapeutic efficacy (15, 16), the strategy of incapacitating or reducing the synovial macrophage population may be effective for the treatment of RA. However, an unbiased and systematic approach has not been undertaken to determine the molecular signatures and biological networks of RA macrophages that underlie the roles of these macrophages in RA pathogenesis. In addition, the role of NFAT5 in the survival 
of RA macrophages has not yet been documented, even though it is known that NFAT5 supports the expression of a group of key proinflammatory genes $(2,5,17)$.

A number of innate and adaptive immune cells interact via an array of cytokines and/or cell-to-cell contacts, generating a complex inflammatory network within the RA synovia $(6,18)$. This complexity presents challenges when trying to determine the specific roles of synovial macrophages in the progression of RA. Comparative and unbiased analyses of gene expression profiles in a single cell population freshly isolated from pathological sites may help to identify the predominant signatures of a certain type of cells involved in RA pathogenesis (19). Here, we performed global transcriptomic profiling of synovial macrophages isolated from RA patients and of NFAT5-deficient macrophages. Analysis of the transcriptomes in the 2 types of macrophages revealed that NFAT5 was a central regulator for diverse pathological processes of RA macrophages, including the cell cycle, apoptosis, and proliferation. Very interestingly, NFAT5 showed a more significant overlap between its regulon and differentially expressed genes (DEGs) in RA synovial macrophages than did TP53 and NF- $\kappa B$ complexes. Proinflammatory M1-polarizing stimuli and hypoxic conditions were responsible for enhanced NFAT5 expression in RA macrophages. Functional studies in vitro demonstrated that NFAT5 promoted survival and prevented apoptosis by inducing CCL2 production in both human and murine macrophages. Moreover, in mice, NFAT5-deficient macrophages were less efficient in promoting joint damage than were NFAT5-sufficient macrophages when injected intra-articularly. Taken together, it can be suggested that NFAT5 regulates macrophage survival and thereby plays a major role in the progression of RA.

\section{Results}

Transcriptomic analysis of RA macrophages reveals NFAT5 to be a key TF. One of the most recognized features of RA is an increased number of synovial macrophages in the intimal and subintimal compartments (8) and that the number of these synovial macrophages correlates with RA activity and severity $(10,11)$. Also, macrophage depletion brings a resolution of chronic arthritis in both mouse and human systems $(20,21)$. However, a systematic analysis of the TFs or regulators as possible drivers of RA macrophage pathology has not been done. To address this, we first profiled the global expression of genes in synovial fluid macrophages from RA patients (RA-SF macrophages) and normal macrophages differentiated from peripheral $\mathrm{CD} 14^{+}$monocytes using the Illumina HumanRef-8 v3.0 microarray. As a result, we identified 1,913 DEGs comprising 1,015 upregulated and 898 downregulated genes with an FDR of less than 0.05 and a fold change of greater than 1.5 between RA-SF macrophages and normal macrophages using an integrative statistical method (Figure 1A). Subsequently, we investigated key TFs responsible for DEGs in RA-SF macrophages using a master regulator analysis algorithm (see Methods and Supplemental Methods). The algorithm computed the statistical significance of the overlap between the regulon of each TF and the DEGs in RA-SF macrophages. Among the $259 \mathrm{TFs}$, we identified 19 key TFs that had a $P$ value of less than 0.05. Figure $1 \mathrm{~B}$ shows the top 10 TFs including SP1, SPI1, NFAT5, NF- $\kappa \mathrm{B}$ complexes (REL, RELB, RELA, and NFKB2), PPARG, TP53, and E2F4, which were ranked on the basis of their $P$ values. Surprisingly, NFAT5 showed a more significant overlap between its regulon and DEGs in RA-SF macrophages than did TP53 and NF- $\kappa$ B complexes, suggesting that NFAT5 may be at least as significant as TP53 and NF- $\kappa \mathrm{B}$ in regulating RA macrophage pathology.

To predict the pathologic significance of NFAT5 as a key TF in RA-SF macrophages, we compared the 1,913 DEGs in RA-SF macrophages with previously published data on RA patients' synovial fibroblasts (RA-FLS) and HUVECs perturbed by NFAT5 downregulation via siRNA (22). Among the 1,493 NFAT5 target genes identified in RA-FLS and HUVECs, 302 DEGs overlapped with the 1,913 DEGs found in RA-SF macrophages $(P<0.00001)$ (Figure 1C). We further analyzed cellular processes enriched by the DEGs in RA-SF macrophages. Functional enrichment analysis revealed that leukocyte proliferation, cell-cycle regulation, apoptosis, cell death, actin cytoskeletal organization, and response to ROS were significantly enriched $(P<0.01)$ by the 1,015 DEGs upregulated in RA-SF macrophages (Figure 1D). Interestingly, the DEGs involved in the cell-cycle regulation, apoptosis, cell death, and proliferation overlapped with more than $25 \%$ of the NFAT5 target genes (blue bars in Figure 1D), suggesting that these are predominant cellular processes represented by the DEGs in RA macrophages and that NFAT5 controls the survival and proliferation of macrophages in patients with RA.

Indeed, NFAT5 expression was higher in RA-SF macrophages than in normal macrophages differentiated from peripheral $\mathrm{CD} 14^{+}$cells, as determined by quantitative real-time PCR (qRT-PCR), Western blotting, and flow cytometry (Figure 1E). We sought to determine which factors contribute to the enhanced NFAT5 expression in RA-SF macrophages. RA-SF macrophages are exposed to a number of proinflammatory cytokines, growth factors, and hypoxia (6). As shown in Figure 2A, macrophage-CSF (M-CSF), IL-1 $\beta$, and the $\mathrm{CoCl}_{2}$ hypoxic stimulus increased NFAT5 expression in peripheral $\mathrm{CD} 14^{+}$cells isolated from mononuclear cells of healthy controls. The M-CSF-induced increase in NFAT5 expression was related to the differentiation of monocytes into the M1 macrophage subset, since M1-polarizing stimuli (IFN- $\gamma$ plus LPS), but not the M2-polarizing cytokine IL-4, synergistically increased NFAT5 expression in M-CSF-stimulated CD14 ${ }^{+}$cells (Figure 2B). Moreover, NFAT5 expression in RA macrophages also increased in response to the hypoxic stimulus $\mathrm{CoCl}_{2}$, hypoxia chamber treatment $\left(\mathrm{O}_{2}<1 \%\right)$, or TLR4 ligation with LPS as determined by Western blotting and flow cytometry (Figure 2, C and D). Together, these data suggest that the proinflammatory cytokine IL-1 $\beta$ and M1-polarizing stimuli are responsible for NFAT5 upregulation in the course of differentiation of monocytes into macrophages in inflamed RA joints and that after differentiation, hypoxic conditions can further increase NFAT5 expression in activated macrophages.

NFAT5-regulated transcriptomes in macrophages. Given the microarray data analysis of RA macrophages (Figure 1, A-D), we wanted to test whether NFAT5 is essential for macrophage survival, apoptosis, and proliferation. To this end and for a better understanding of the role for NFAT5 in macrophage biology, we performed transcriptomic analysis of RAW 264.7 macrophages stably deficient in NFAT5 expression. To identify more reliable NFAT5 transcriptional targets, we used 2 strategies: a) stable 
A

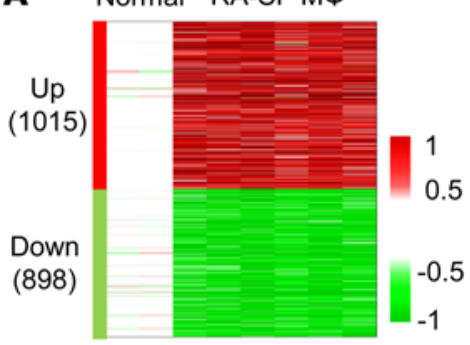

B

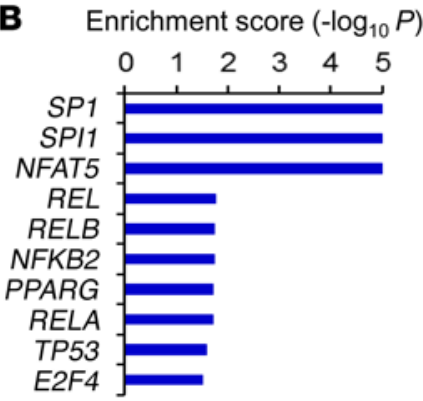

C RA-SF NFAT5

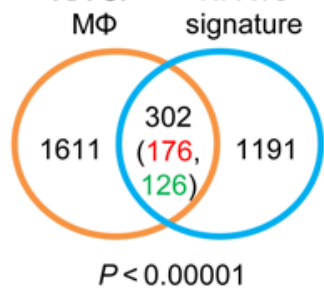

D

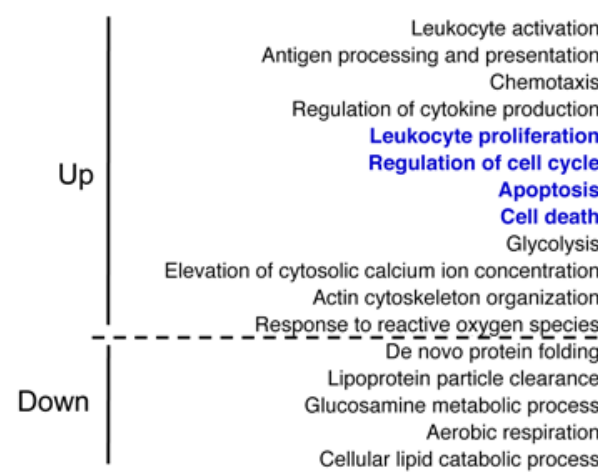

$\%$ of overlap

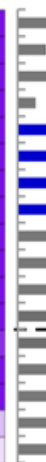

E

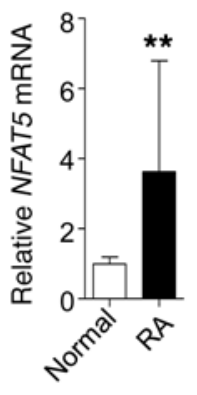

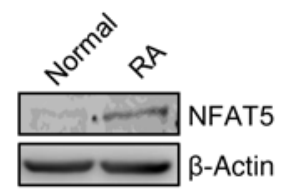

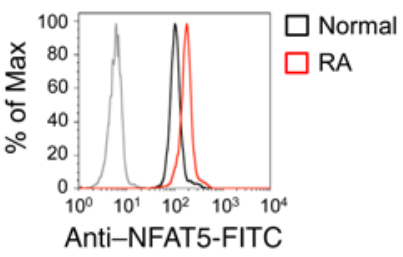

Enrichment score $\left(-\log _{10} P\right)$

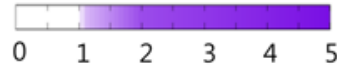

Figure 1. Enhanced NFAT5 signature in RA synovial macrophages. (A) Heatmap displaying the differential expression patterns of 1,913 DEGs in CD14 macrophages from RA-SF $(n=6)$ compared with normal macrophages $(n=2)$ differentiated from peripheral CD14+ monocytes. Red and green colors denote upregulation and downregulation, respectively. M $\varphi$, macrophages. (B) Top 10 TFs enriched by up- and downregulated DEGs in RA synovial macrophages. (C) Venn diagram depicting the overlap between DEGs in synovial macrophages from RA patients and those in NFAT5-deficient RA synovial fibroblasts and human endothelial cells. $P$ value indicates the significance of 302 overlapping DEGs, including 176 upregulated and 126 downregulated DEGs. Empirical hypothesis testing based on 100,000 random permutations was performed to determine the $P$ value of the overlapping DEGs. (D) Cellular processes enriched by the DEGs in synovial macrophages from RA patients. Heatmap with violet color gradient represents the level of significance for each cellular process. Dark violet and bright violet refer to high $(P<0.01)$ and moderate $(P<0.05)$ enrichment, respectivel. The $P$ values were determined by DAVID software using a hypergeometric test method. The bar graph on the right depicts the percentages of overlap between the DEGs associated with each cellular process and NFAT5 target genes. (E) Enhanced NFAT5 expression in RA synovial macrophages. Synovial macrophages were freshly isolated from synovial fluid of RA patients using anti-CD14 magnetic beads. Normal macrophages were differentiated from peripheral monocytes of healthy subjects. NFAT5 expression was determined by real-time PCR $(n=9)$, Western blot, and flow cytometric analyses. The gray plot in the flow cytometric graph indicates the isotype control in normal macrophages. NFAT5 mRNA expression is shown as a the fold increase relative to GAPDH mRNA. Data represent the mean \pm SD. ${ }^{* *} P<0.005$ versus normal macrophages, by Mann-Whitney $U$ test.

knockdown (NFAT5 KD) of Nfat5 transcripts in RAW 264.7 cells using shRNA for Nfat5; and b) a decoy system (NFAT5 decoy) that inhibits NFAT5 binding to its promoter by stable transduction of the cells with oligonucleotides harboring the consensus sequence for NFAT5 target genes (23). By an integrative statistical method, we identified 2,205 and 2,014 DEGs in RAW 264.7 macrophages with NFAT5 KD and NFAT5 decoy, respectively (Figure 3A). Comparison of the DEGs showed that $87 \%$ of the genes were in common to both NFAT5-KD and NFAT5 decoy cells, indicating that our approach to repressing transcriptional activity of NFAT5 was reliable. We then selected 1,893 of the 1,958 genes that were regulated in the same direction (up- or downregulated) as transcriptional targets of NFAT5 in both conditions (Figure 3B). Cellular processes represented by the 1,893 genes were then categorized into 9 functional modules, which included translation, cell prolif- eration, DNA repair, RNA processing, mitochondrial processes, epigenetic regulation, transport, cell death, and ER stress (Figure 3C). Of note, cell proliferation and cell death were two of the most significant biologic processes functionally enriched by the DEGs, as determined by their enrichment scores (Figure 3C), suggesting that these are the predominant cellular processes regulated by NFAT5 in macrophages.

We focused on macrophage survival and proliferation as the major biological processes regulated by NFAT5. Biological networks delineating key cellular processes are essential to understanding the function of a gene at the molecular level (19). Thus, we integrated NFAT5-dependent modules involving DNA repair, cell-cycle regulation, survival, death, and proliferation and then reconstructed a molecular network representing these processes (Figure 3D). Next, we selected 10 representative genes from NFAT5-regulated DEGs 
A

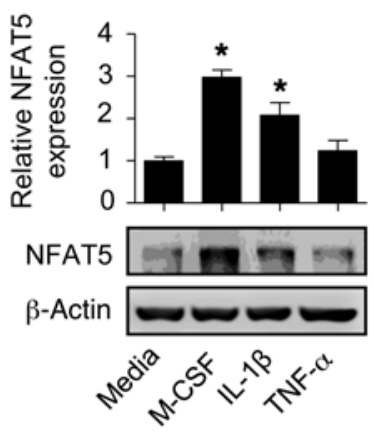

B

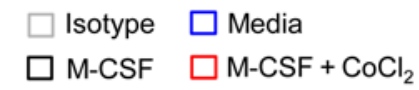

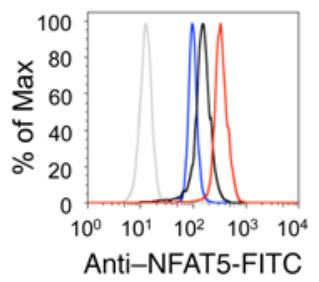

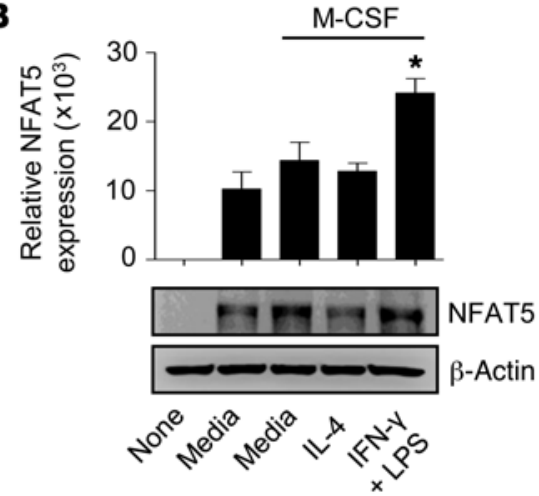

C

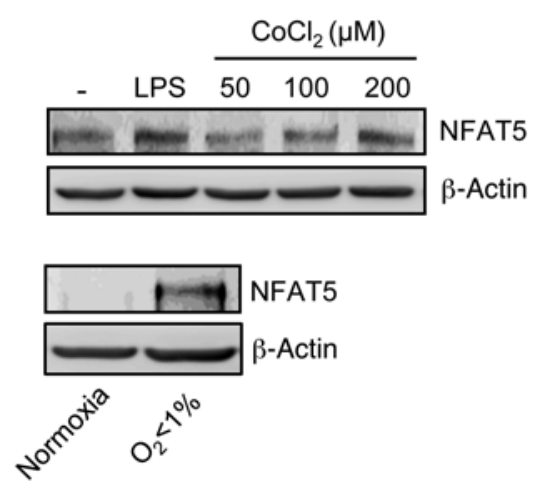

D

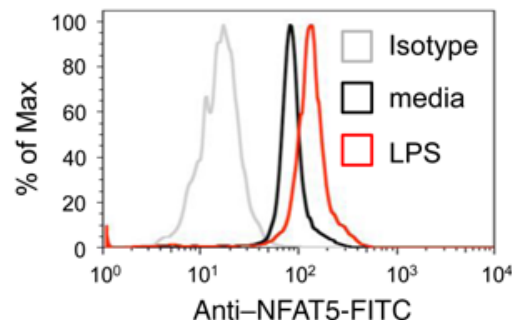

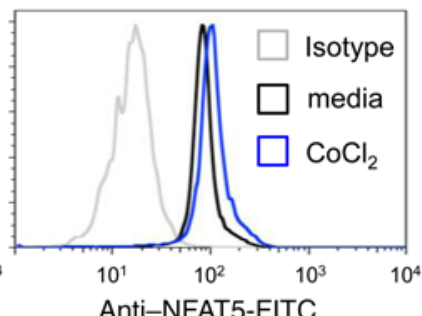

Figure 2. Induction of NFAT5 expression in macrophages by M1 polarizing and hypoxic stimuli. (A) Increase in NFAT5 expression by IL-1 1 , M-CSF, and CoCl

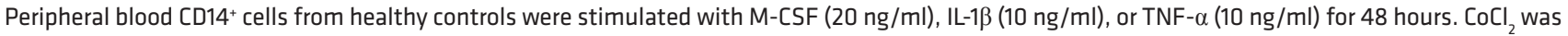
treated again for 6 hours after stimulation with M-CSF. NFAT5 expression was determined by Western blot and flow cytometric analyses. Data represent the mean $\pm S D$ and are representative of 3 independent experiments. ${ }^{*} P<0.05$ versus media only, by Mann-Whitney $U$ test. (B) Increase in NFAT5 expression by M1-polarizing stimuli. Normal peripheral monocytes (CD14+ cells) were stimulated with M-CSF $(20 \mathrm{ng} / \mathrm{ml})$ for 48 hours and then stimulated again with IL-4 $(20 \mathrm{ng} / \mathrm{ml})$ or IFN- $\gamma(20 \mathrm{ng} / \mathrm{ml})$ plus LPS $(100 \mathrm{ng} / \mathrm{ml})$. NFAT5 expression was determined by Western blot analysis. Data represent the mean \pm SD of 3 independent experiments. ${ }^{*} P<0.05$ versus M-CSF only, by Mann-Whitney $U$ test. (C and $\mathbf{D}$ ) Hypoxia-induced increase in NFAT5 expression in RA synovial macrophages. CD14+ macrophages were freshly isolated from RA-SF and stimulated with $\mathrm{CoCl}_{2}$ or in the hypoxia chamber $\left(\mathrm{O}_{2}<1 \%\right)$. NFAT5 expression was determined by Western blot analysis (C) after 24 hours and flow cytometric analysis (D) after 6 hours. Data are representative of 3 separate experiments.

and validated their differential expression using qRT-PCR. As shown in Figure 3E and Supplemental Table 1 (supplemental material available online with this article; doi:10.1172/JCI87880DS1), the expression levels of Cd74, Sod2, Ccl2, Bop1, Cdkn2d, and Ran mRNA, which are involved in cell-cycle regulation, survival, and proliferation (24-28), were decreased in NFAT5-deficient RAW 264.7 cells when compared with levels in control vector-transfected cells. In contrast, the expression levels of Dap3, Fas, Stk3, and Tiam1 mRNA, which promote apoptotic death $(13,29-31)$, were increased in the same cells. Moreover, the same relative changes in RNA message levels were observed in NFAT5-deficient (via shRNA) CD14 ${ }^{+}$ macrophages from human subjects as those in NFAT5-deficient RAW 264.7 cells (Figure 3E and Supplemental Table 1). Collectively, these data support the view that NFAT5 may control the survival and proliferation of macrophages.

NFAT5 is required for macrophage survival and proliferation. To verify the hypothesis on NFAT5 regulation of macrophage survival and proliferation, we performed flow cytometric analysis and an MTT (tetrazolium) assay of NFAT5-deficient RAW 264.7 macrophages. As shown in Figure 4A, NFAT5-KD RAW 264.7 macrophages treated with cycloheximide (CHX) for 24 hours showed an increase in the frequency of FITC-annexin $\mathrm{V}^{+}$and/or propidium iodide ${ }^{+}\left(\mathrm{PI}^{+}\right)$cells on flow cytometric analysis as com- pared with the control cells. The frequency of annexin $\mathrm{V}^{+}$and/or $\mathrm{PI}^{+}$cells induced by thapsigargin (TG) and sodium nitroprusside (SNP) was also higher in NFAT5-KD RAW 264.7 macrophages than in control vector-transfected cells (Figure 4B), indicating that NFAT5 deficiency renders RAW 264.7 macrophages more susceptible to TG- or SNP-induced apoptosis. In parallel, when treated with SNP, NFAT5-KD RAW 264.7 macrophages showed a decrease in antiapoptotic BCL-2 expression and an increase in proapoptotic caspase 8 expression compared with expression levels seen in controls cells (Supplemental Figure 1A). The starvation-, CHX-, or TG-induced increase in cleaved caspase 8 expression was also greater in NFAT5-KD cells than in control cells (Supplemental Figure 1, B-D). Moreover, cell viability, as determined by MTT assay, was significantly lower in NFAT5-deficient RAW 264.7 macrophages (in both NFAT5-KD and NFAT5 decoy cells) in the presence of CHX, SNP, or TG than in control cells (Figure 4C), indicating that NFAT5 controls the survival of RAW 264.7 macrophages and that, with reduced NFAT5 activity, they are more susceptible to apoptosis.

Compared with control cells, the number of viable cells assessed by trypan blue exclusion was significantly reduced for NFAT5-deficient RAW 264.7 macrophages, with a reduction of approximately $25 \%$ to $30 \%$ over a 96 -hour culture period (Figure $4 \mathrm{D}$ ). The prolifer- 
A

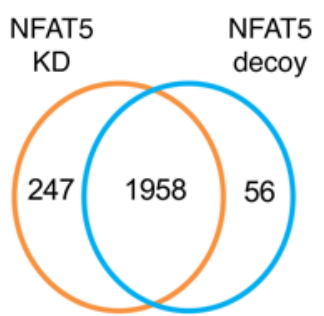

B

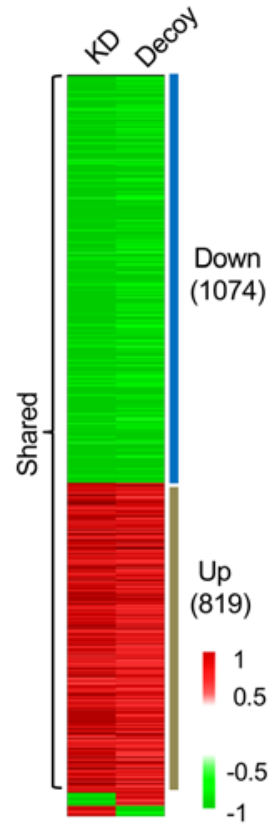

E

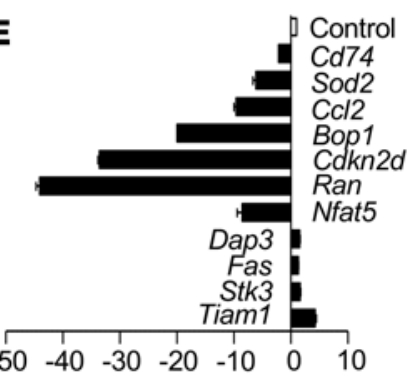

Relative mRNA expression

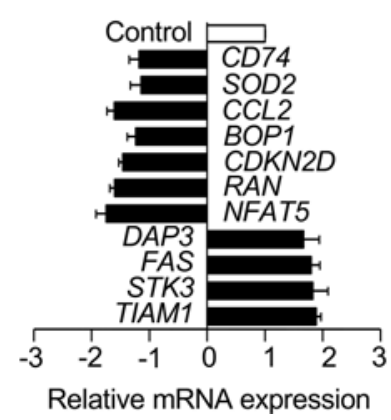

C
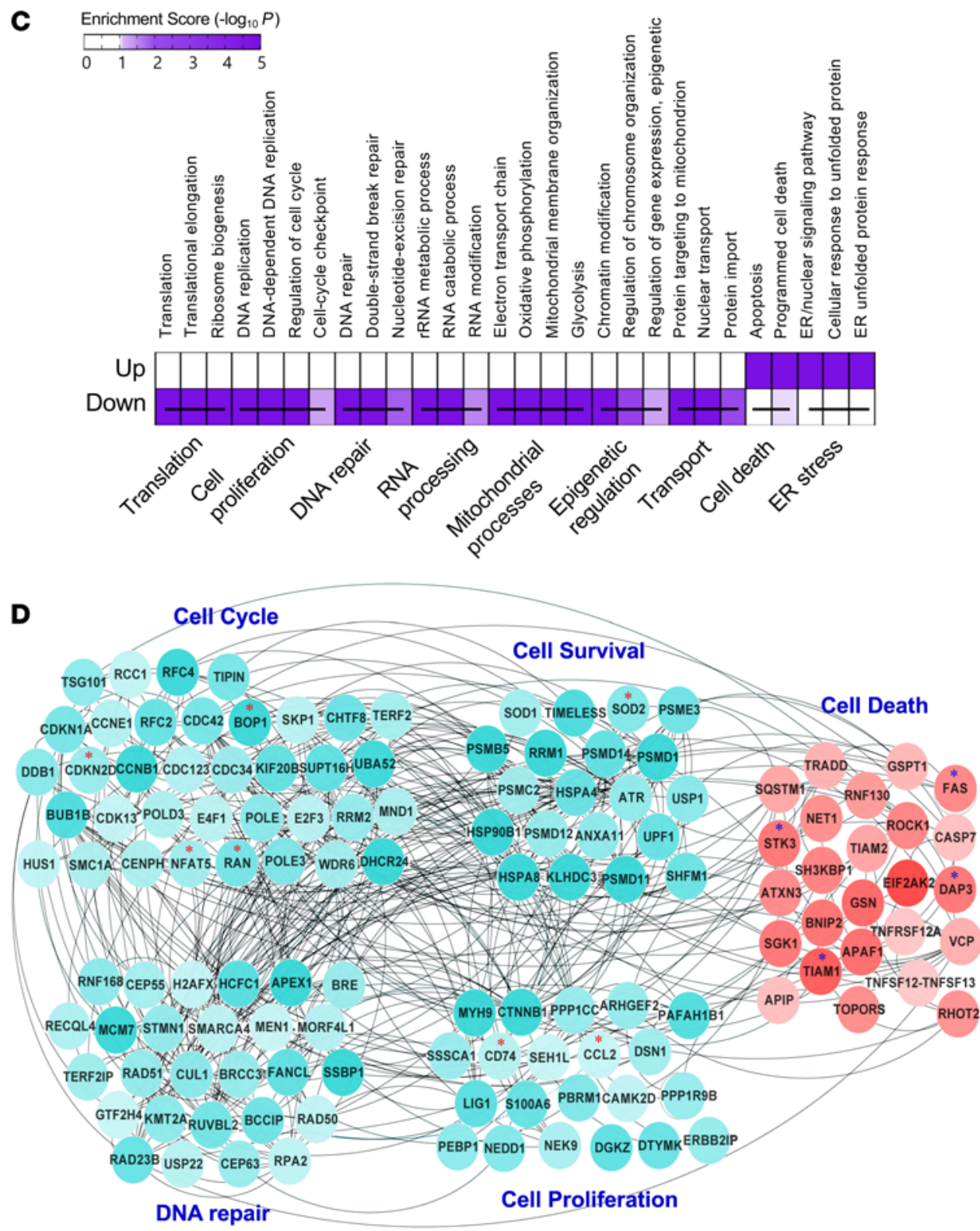

Figure 3. Molecular targets of NFAT5 involving RAW 264.7 macrophage survival and proliferation. (A-C) Comparative analysis of the transcriptomes in RAW 264.7 macrophages with NFAT5 shRNA $(n=3)$, control shRNA $(n=3)$, and NFAT5 decoy oligonucleotides $(n=3)$. Global gene expressions profiles were determined using the Illumina MouseWG-6 v2.0 array platform. (A) Venn diagram illustrates the overlap between DEGs in macrophages stably transfected with NFAT5 shRNA (NFAT5 KD) and those transduced stably with NFAT5 decoy oligonucleotides (TGGAAAATTACCG, NFAT5 decoy). (B) Heatmap showing differential expression patterns of 2,261 DEGs in NFAT5 KD and the NFAT5 decoy compared with the corresponding controls. (C) Cellular processes enriched by 1,074 (Down) and 819 (Up) DEGs in the same direction between NFAT5 KD and the NFAT5 decoy. Significantly enriched processes for common DEGs were scored with respect to the $P$ value obtained. Selected processes were grouped into 9 functional classes on the basis of the similarity of gene ontology terms defined by the kappa score. The color gradient on the right represents the enrichment score defined as $-\log _{10}(P$ value $)$. Enrichment scores are displayed in a violet color gradient: dark violet $(P<0.01)$, bright violet $(P<0.05)$, and white $(P>0.05)$. The significance of the enrichment was determined using a hypergeometric test. (D) Network model describing the functional modules, which are functionally aggregated with the DEGs associated with cell proliferation, apoptosis, and survival. Node color represents upregulated (red) and downregulated (turquoise) gene expression in NFAT5 KD and the NFAT5 decoy compared with control. Gray lines indicate interactions between 2 genes. Expression levels of the genes marked with an asterisk were confirmed by real-time PCR in Figure 3E. (E) mRNA expression levels of representative NFAT5-regulated genes involved in cell survival, proliferation, and apoptosis in NFAT5 KD RAW 264.7 macrophages (upper panel) and NFAT5 shRNA-transfected human macrophages (lower panel), as determined by real-time PCR. Data represent the mean \pm SD of 3 independent experiments performed in duplicate. 
A

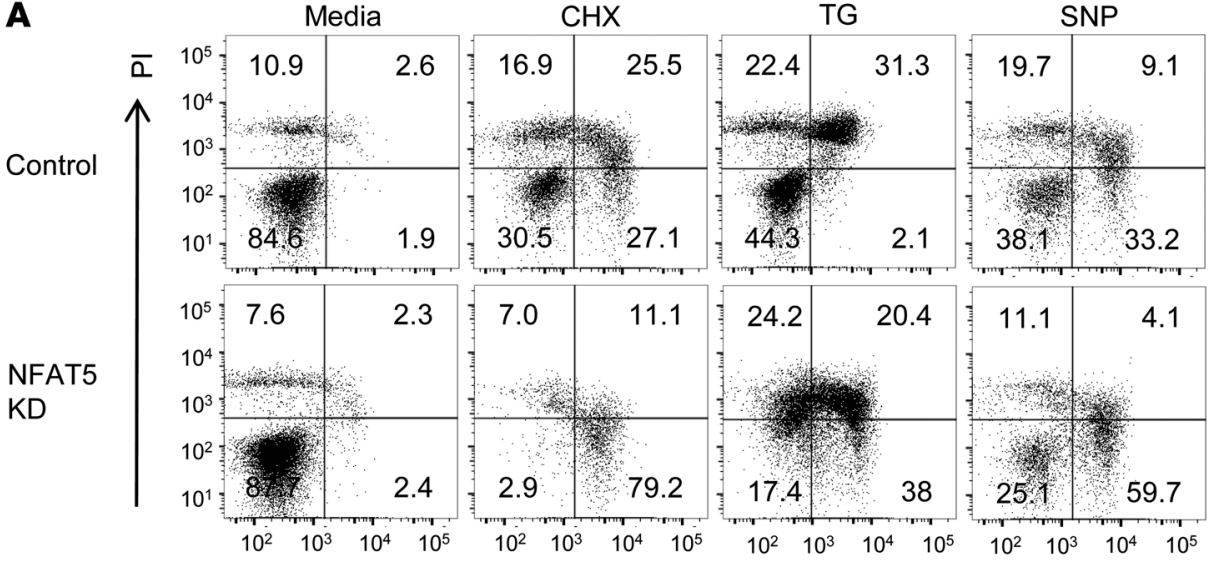

B

NFAT5 KD

$\square$ Control
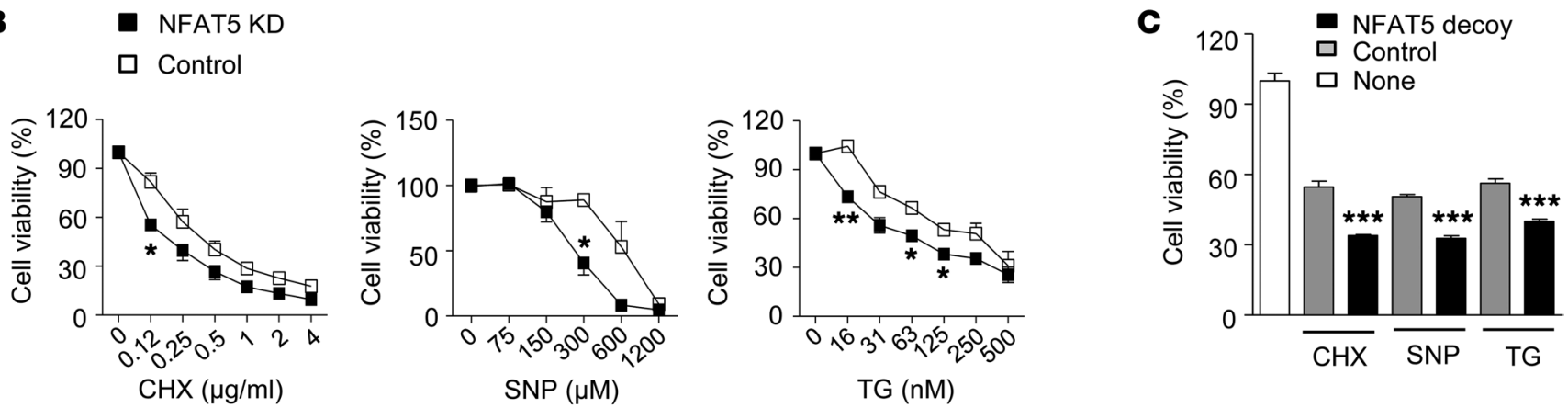

D

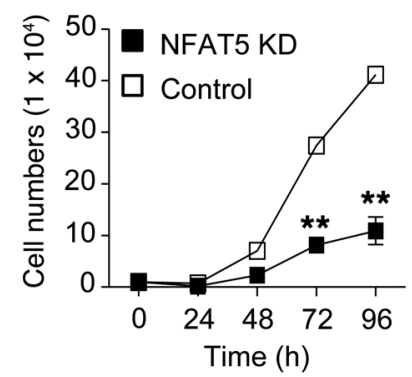

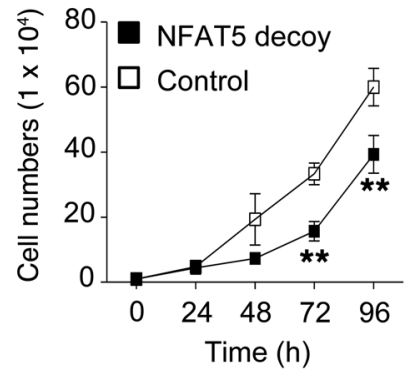

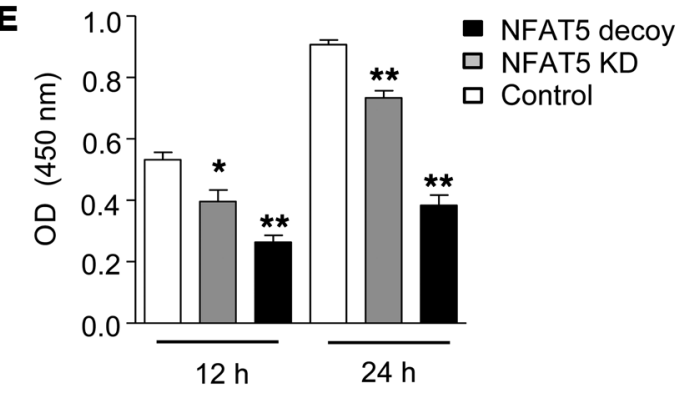

Figure 4. NFAT5 regulates the survival and proliferation of RAW 264.7 macrophages. (A) Increased apoptotic death in NFAT5-deficient RAW 264.7 macrophages. The degree of apoptosis was assessed in NFAT5 shRNA-transfected (NFAT5 KD) RAW 264.7 macrophages versus control vector-transfected cells (Control) 24 hours after treatment with CHX $(0.5 \mu \mathrm{g} / \mathrm{ml})$, TC $(100 \mathrm{nM})$, or SNP $(100 \mu \mathrm{M})$ using flow cytometry for FITC-annexin V and PI. Data are representative of 3 independent experiments. (B and C) Decreased viability of NFAT5-deficient RAW 264.7 macrophages. NFAT5 KD RAW 264.7 macrophages $\left(1 \times 10^{5}\right.$ cells $)$ were treated with CHX $(0-4 \mu \mathrm{g} / \mathrm{ml})$, SNP $(0-1,200 \mu \mathrm{M})$, or TC $(0-500 \mathrm{nM})$ for 24 hours. RAW 264.7 macrophages stably transduced with the NFAT5 decoy were treated with $\mathrm{CHX}(0.5 \mu \mathrm{g} / \mathrm{ml})$, SNP $(1.2 \mathrm{mM})$, or TC $(16 \mathrm{nM})$ for 24 hours. Cell viability was determined by MTT assay. Data represent the mean \pm SD of 3 independent experiments. ${ }^{*} P<0.05$, ${ }^{* *} P<0.01$, and ${ }^{* * *} P<0.001$ versus control vector-transfected RAW 264.7 cells, by 1 -way ANOVA with Tukey's post-hoc test. ( $D$ and $\mathbf{E})$ Reduction of RAW 264.7 cell proliferation due to NFAT5 deficiency. RAW 264.7 macrophages $\left(1 \times 10^{4}\right.$ cells) stably transfected with NFAT5 shRNA (NFAT5 KD) or stably transduced with NFAT5 decoy were cultured for 96 hours in RPMI 1640 containing $10 \%$ FBS. Viable cells were manually counted after trypan blue staining (D). Cell proliferation was determined by BrdU assay following a 12- or 24-hour incubation with $10 \%$ FBS (E). Data represent the mean \pm SD. ${ }^{*} P<0.05$ and ${ }^{* *} P<0.001$ versus control vector-transfected cells, by 1-way ANOVA with Tukey's post-hoc test.

ation of RAW 264.7 macrophages was decreased in the NFAT5-KD or the NFAT5 decoy cells, as determined by a BrdU incorporation assay (Figure 4E), suggesting that NFAT5 deficiency reduced the proliferation of RAW 264.7 macrophages.

To demonstrate whether primary macrophages are controlled by NFAT5 in a manner similar to that seen with RAW 264.7 macrophages, we investigated the effect of NFAT5 deficiency on the apoptosis and survival of murine peritoneal macrophages. Since complete NFAT5 deficiency is developmentally lethal, we used a line of mice heterozygous for NFAT5 [referred to hereafter as $\mathrm{Nfat} 5^{+/-}$mice] with an inactive allele (32). The heterozygotes displayed haploinsufficiency of Nfat 5 mRNA and protein expression in many tissues and cells, including macrophages (data not shown and ref. 32). As expected, apoptotic cell death induced by $\mathrm{CHX}$, SNP, or TG, as assessed by follow cytometry, was substantially higher in $\mathrm{Nfat}^{+/-}$macrophages than in WT $\left(\mathrm{Nfat}^{+/+}\right)$macrophages 
A

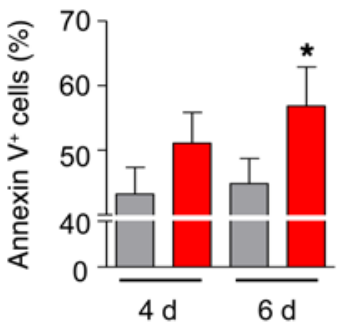

Time (d)

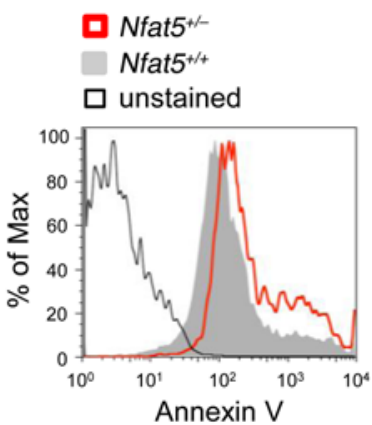

D

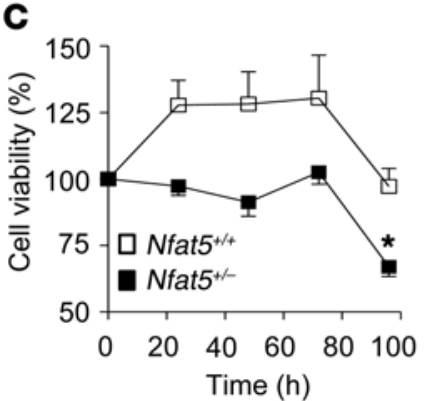

B

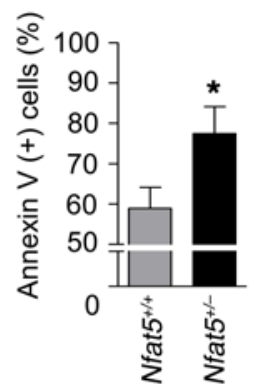

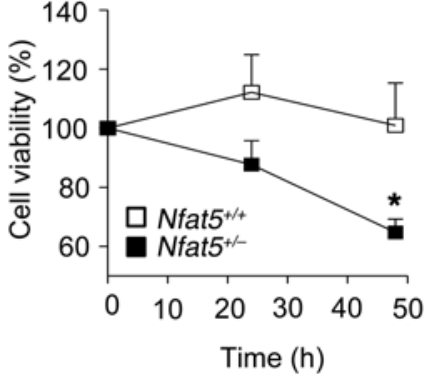
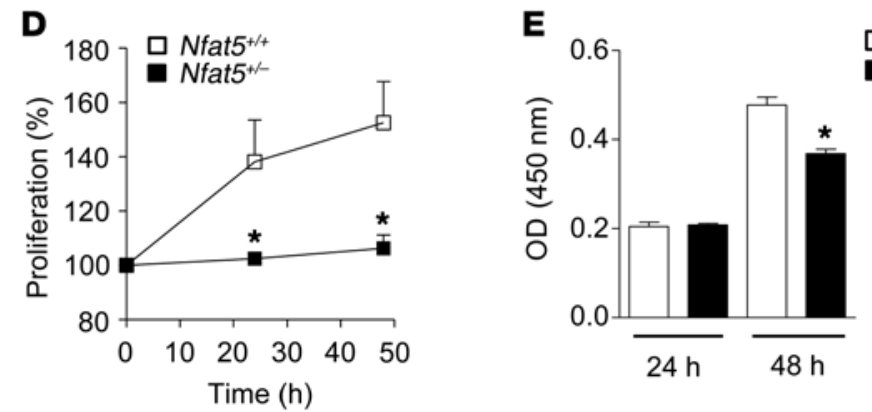

Nfat5 ${ }^{+/+}$

Nfat5 $^{+/-}$

Figure 5. Decrease in survival and proliferation of primary mouse macrophages due to NFAT5 deficiency. (A and B) Increased apoptosis in primary macrophages due to NFAT5 haploinsufficiency. Splenic macrophages ( $2 \times 10^{5}$ cells) from WT Nfat5 $5^{+/+}$and $\mathrm{Nfat5}^{+/-}$mice were obtained 24 hours after i.p. injection of LPS (10 mg/kg) and were cultured in RPMI containing 10\% FBS for 4 and 6 days (A) or in the presence of CoCl 2 (100 $\mu$ M) for 2 days (B). The extent of apoptotic death was determined by flow cytometry for annexin $\mathrm{V}$ and by MTT assay. ${ }^{*} P<0.05$ versus WT Nfat5 ${ }^{+/+}$macrophages, by Mann-Whitney $U$ test. (C) Starvation-induced cell death in $N f a t 5^{+/+}$versus $\mathrm{Nfat}^{+/-}$mice, as determined by MTT assay. ${ }^{*} \mathrm{P}<0.05$ versus $\mathrm{Nfat} 5^{+/+}$macrophages, by Mann-Whitney $U$ test. ( $\mathbf{D}$ and $\mathbf{E}$ ) Decreased proliferation of primary macrophages due to NFAT5 insufficiency. Thioglycollate peritoneal macrophages ( $\times 10^{5}$ cells) from $N$ fat $5^{+/+}$ and $\mathrm{Nfat5}^{+/-}$mice were stimulated with RPMI 1640 containing $10 \%$ FBS. Cell proliferation was determined by MTT assay (D) or BrdU assay (E) after a $24-$ and 48-hour incubation. ${ }^{*} P<0.05$ versus $\mathrm{Nfat}^{+/+}$macrophages, by Mann-Whitney $U$ test. Data in B-E were compiled from 2 experiments ( $n=4$ mice per group).

(Supplemental Figure 2). Moreover, the mean annexin V fluorescence intensity was significantly higher in splenic macrophages from $\mathrm{Nat5}^{+/-}$mice than in those from $\mathrm{Nfat5^{+/+ }}$ mice, as determined 6 days after incubation (Figure 5A). The extent of apoptotic death induced by the $\mathrm{CoCl}_{2}$ hypoxic stimulus was also higher in splenic $\mathrm{Nat5}^{+/-}$macrophages (Figure 5B), confirming that NFAT5 insufficiency promotes apoptosis of primary macrophages. In parallel, macrophage survival under serum-free (starvation) conditions was significantly lower in $\mathrm{Nat5}^{+/-}$macrophages than in $\mathrm{Nfat}^{+/+}$ macrophages, as measured by MTT assay (Figure 5C). Additionally, the number of cells stimulated with 10\% FBS for 48 hours was significantly reduced in $\mathrm{Nat5}^{+/-}$macrophages (Figure 5D). The extent of BrdU incorporation was also lower in $\mathrm{Nat}^{+/-}$macrophages than in $\mathrm{Nat5}^{+/+}$macrophages (Figure 5E), which suggests that NFAT5 is involved in the proliferation of primary mouse macrophages. Moreover, the NFAT5-mediated deficiency in macrophage survival and proliferation was reproduced in bone marrow-derived macrophages (BMDMs) isolated from $\mathrm{Nfat}^{+/-}$mice (Supplemental Figure 3). Taken together, these results demonstrate that NFAT5 is required for the survival and proliferation of RAW 264.7 and primary macrophages.

The NFAT5 target CCL2 is necessary for RA macrophage survival. We performed our next experiment to validate whether NFAT5 actually controls the survival of RA macrophages and, if so, to determine which target genes of NFAT5 are predominantly involved in this process. To this end, we first performed qRT-PCR analysis for the representative NFAT5-regulated genes shown in Figure 3E in independent RA-SF macrophage samples. In sharp contrast to NFAT5-deficient conditions, CD74, SOD2, and CCL2 mRNA expression was increased in RA-SF macrophages, whereas APAF1, DAP3, STK3, and FAS mRNA expression was decreased in the same cells (Figure 6A). In particular, CCL2 was the most highly expressed gene among the 10 NFAT5 target genes in RA-SF macrophages (Figure 6A), with a 76.3-fold higher mean level of CCL2 mRNA detected in RA macrophages than in normal macrophages.

CCL2 promotes the survival of human $\mathrm{CD} 11 \mathrm{~b}^{+}$peripheral blood mononuclear cells (PBMCs) (24). To predict the role of CCL2 in the survival of RA macrophages, we reconstructed a CCL2-centered network using the DEGs in RA-SF macrophages. Supplemental Figure 4A and Supplemental Table 2 illustrate significant interactions among the first neighbors of CCL2 that are associated with cell proliferation, survival, and antiapoptosis $(P<0.00001)$, which suggests a CCL2-dependent regulation of RA macrophage survival. In support of this observation, expression of CD44 and OLR1, the first neighbors of CCL2, was significantly higher in CCL2 ${ }^{+}$macrophages than in CCL2 ${ }^{-}$macrophages in RA-SF, as determined by flow cytometry (Supplemental Figure $4 \mathrm{~B})$. Thus, we postulated that CCL2 is secreted by RA macrophages in an NFAT5-dependent manner and contributes to RA macrophage survival in an autocrine and paracrine manner. 
A

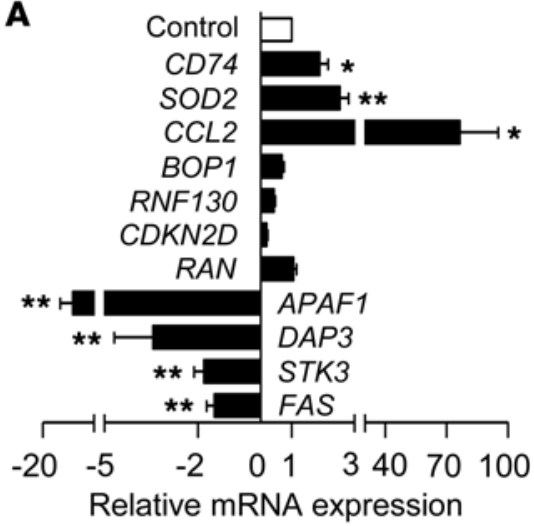

D
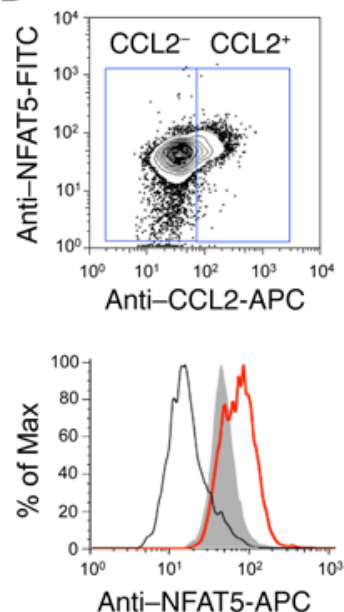

B

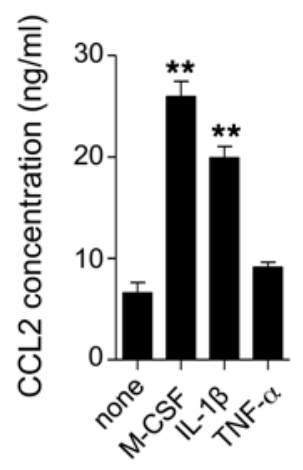

C

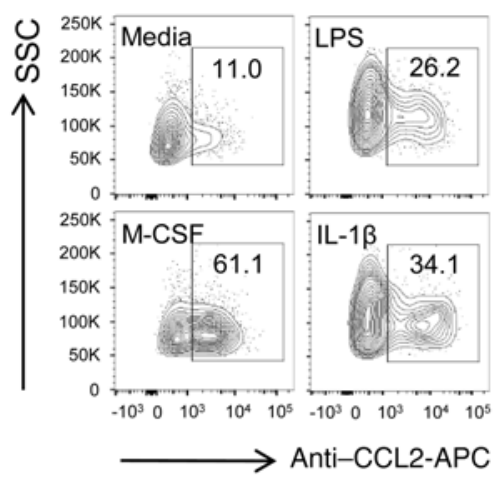

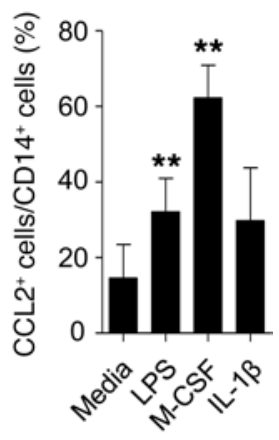

$\mathbf{F}$

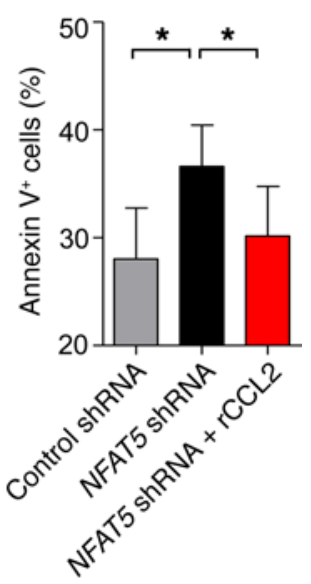

Figure 6. The NFAT5 target CCL2 prevents apoptotic death of RA macrophages. (A) mRNA expression levels of representative NFAT5-regulated genes involved in cell survival, proliferation, and apoptosis in RA-SF macrophages $(n=8)$ compared with normal macrophages ( $n=8$ ), as determined by real-time PCR and presented according to fold change. (B and C) Increased CCL2 expression in CD14+ cells in response to NFAT5-inducing stimuli. Peripheral blood

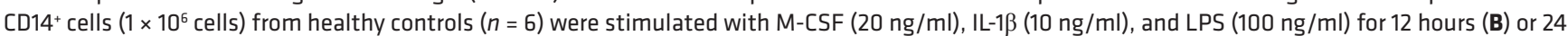
hours (C). CCL2 expression in the culture supernatants was determined by ELISA (B) and intracellular flow cytometry (C). (D) NFAT5 expression in CCL2 ${ }^{+}$ versus CCL2- subpopulations of RA-SF macrophages $(n=3)$, as determined by flow cytometry. Gates for the CCL2+ or CCL2- ${ }^{-}$cells are shown in the flow cytometric dot plot. Histogram shows the difference in NFAT5 expression according to CCL2 positivity. MFI, mean fluorescence intensity. (E) Decreased CCL2 secretion by NFAT5-deficient human macrophages. Peripheral CD14+ cells $\left(1 \times 10^{6}\right.$ cells, $\left.n=4\right)$ were stimulated with M-CSF for 24 hours and then transfected with NFAT5 shRNA or control shRNA for a further 24 hours. CCL2 levels in the culture supernatants were measured by ELISA (bar graph) and were also assessed by flow cytometry (flow cytometric dot plot) in macrophages 24 hours after stimulation with 10 ng/ml LPS. (F) Effect of CCL2 on NFAT5-dependent apoptosis of RA-SF macrophages. RA-SF macrophages ( $2 \times 10^{5}$ cells) were transfected with NFAT5 shRNA or control shRNA for 24 hours. The cells were then cultured in the presence or absence of $100 \mathrm{ng} / \mathrm{ml}$ recombinant CCL2 (rCCL2) $(n=5)$ for 24 hours. The extent of apoptosis was determined by flow cytometry for annexin V. Bar graphs in $\mathbf{A}-\mathbf{F}$ represent the mean $\pm \mathrm{SD}$. (A) ${ }^{*} P<0.05$ and ${ }^{*} P<0.001$ versus normal macrophages, by Mann-Whitney $U$ test. (B and $\mathbf{C}$ ) ${ }^{* *} P<0.001$ versus media only, by 1-way ANOVA with Tukey's post-hoc test. (D, E, and $\mathbf{F}$ ) ${ }^{*} P<0.05$ between groups, by Mann-Whitney $U$ test. FSC, forward scatter; SSC, side scatter.

To explore this hypothesis, we first determined CCL2 expression levels in human monocytes stimulated with proinflammatory cytokines and LPS. As reported previously $(2,22)$, we found that NFAT5-inducing stimuli, including M-CSF, IL-1 $\beta$, and LPS, could upregulate the expression of CCL2 in normal peripheral $\mathrm{CD} 14^{+}$cells as determined by ELISA and flow cytometry (Figure $6, \mathrm{~B}$ and $\mathrm{C}$ ). Interestingly, CCL2 ${ }^{+} \mathrm{RA}$ macrophages had higher levels of NFAT5 than did CCL2- RA macrophages (Figure 6D). Double immunofluorescence staining of RA synovium also revealed the coexpression of NFAT5 and CCL 2 in CD14 ${ }^{+}$macrophages, however, NFAT5 ${ }^{-}$macrophages only weakly expressed CCL2 (Supplemental Figure 5). Moreover, NFAF5 KD with shRNAs using a lentiviral system resulted in a significant decrease in CCL2 secretion by normal macrophages (Figure 6E); a reduction of CCL2 secretion was also seen in NFAT5-deficient RAW 264.7 macrophages (Supplemental Figure 6A). Collectively, these observations suggest that CCL2 is produced by RA macrophages via an NFAT5-dependent pathway upon TLR4 ligation and proinflammatory cytokine stimulation. To investigate whether NFAT5 promotes RA macrophage survival via CCL2 secretion, we performed annexin $\mathrm{V}$ staining of RA-SF macrophages in the presence of exogenous CCL2 after transfection of NFAT5 shRNA using a lentiviral system. As shown in Figure 6F, NFAT5 shRNA significantly increased the mean intensity of annexin $\mathrm{V}^{+}$cells as compared with control (scrambled) shRNA, which was completely recovered by the addition of recombinant CCL2. 
A

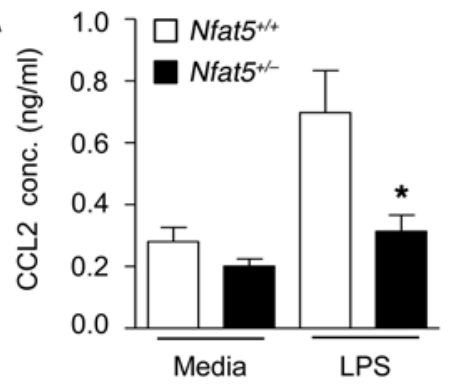

C

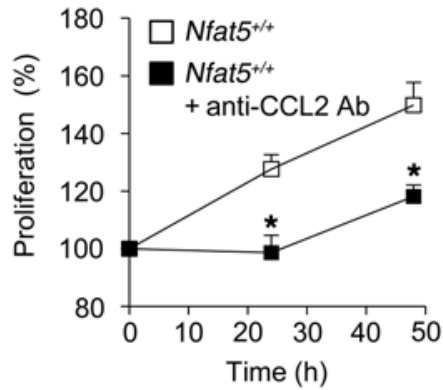

B
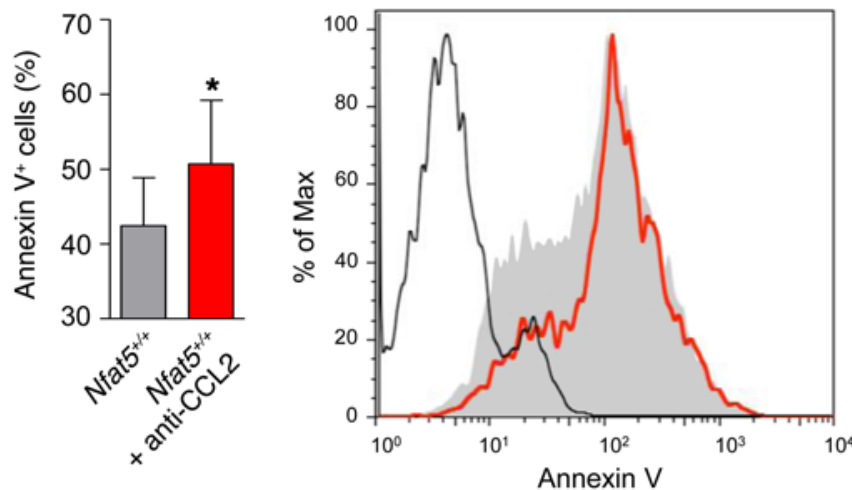

D
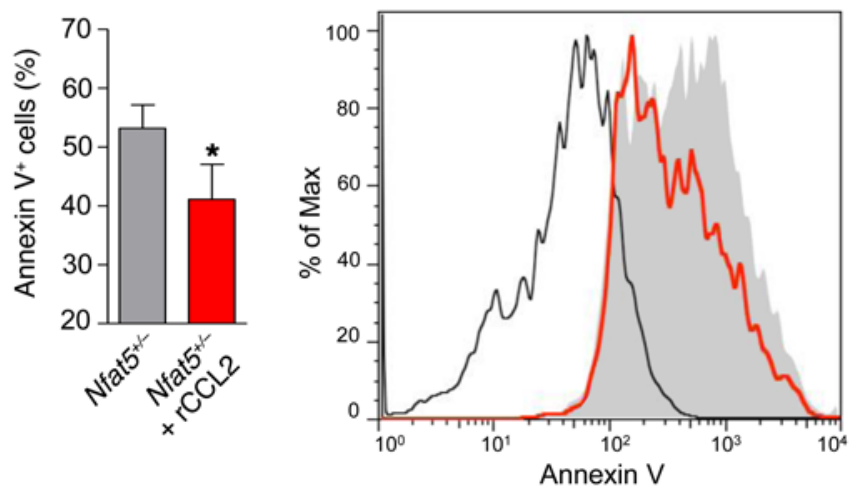

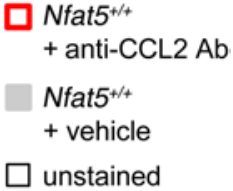

unstained

$\mathrm{Nfat5}^{+/-}$

$+\mathrm{rCCL} 2$

Nfat5

+ vehicle

unstained

Annexin V

Figure 7. NFAT5 promotes the survival of primary mouse macrophages by inducing CCL2 secretion. (A) CCL2 secretion by macrophages from WT $\mathrm{Nfat}^{+/+}$and $\mathrm{Nfat5^{+/- }}$ mice. Peritoneal macrophages $\left(1 \times 10^{5}\right.$ cells) were isolated 96 hours after i.p. injection of thioglycollate and then stimulated with LPS $(100 \mathrm{ng} / \mathrm{ml})$ for 24 hours. CCL2 concentrations in the culture supernatants were measured by ELISA. Data were compiled from 2 experiments $(n=4$ mice per group) and are presented as the mean $\pm S D$. ${ }^{*} P<0.05$, by Mann-Whitney $U$ test. (B) Increased apoptosis of splenic macrophages in response to anti-CCL2 Ab. Splenic macrophages $\left(2 \times 10^{5}\right.$ cells) from Nfat $5^{+/+}$mice were treated with anti-CCL2-neutralizing Ab $(5 \mu \mathrm{g} / \mathrm{ml})$ for $48 \mathrm{hours}$. The degree of apoptosis was determined by flow cytometry for annexin V. Data represent the mean \pm SD of 3 independent experiments ( $n=6$ per group). ${ }^{*} P<0.05$ by Mann-Whitney $U$ test. (C) Reduced proliferation of thioglycollate macrophages in response to anti-CCL2 Ab. Thioglycollate peritoneal macrophages $\left(1 \times 10^{5}\right.$ cells) from $\mathrm{Nfat}^{+/+}$mice were stimulated with $10 \%$ FBS for 48 hours in the presence or absence of anti-CCL2 Ab $(5 \mu \mathrm{g} / \mathrm{ml})$. Cell viability was assessed by MTT assay. Data represent the mean \pm SD of 3 independent experiments ( $n=6$ per group). ${ }^{*} P<0.05$ between groups at each time point, by

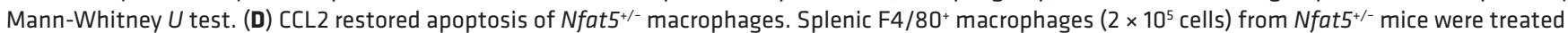
with recombinant CCL2 $(200 \mathrm{ng} / \mathrm{ml})$ for 6 days. Apoptosis was assessed by flow cytometry for annexin V. Data were compiled from 2 experiments $(n=4$ mice per group). ${ }^{*} P<0.05$, by Mann-Whitney $U$ test.

To exclude the off-target effects of shRNA on NFAT5, we used primary macrophages from $\mathrm{Nfat}^{+/-}$mice. We isolated macrophages from splenic cells activated with LPS in vivo or from peritoneal cells stimulated with thioglycollate in vivo and tested whether NFAT5 regulates macrophage survival via CCL2. When stimulated with LPS for 24 hours, $\mathrm{Nfat}^{+/-}$macrophages produced significantly lower levels of CCL2 than did WT $\mathrm{Nfat}^{+/+}$macrophages (Figure 7A). Next, thioglycollate-peritoneal macrophages or splenic $\mathrm{F} 4 / 80^{+}$macrophages from $\mathrm{Nfat}^{+/+}$mice were exposed to anti-CCL2-neutralizing Ab, and their cell viability was examined. The results showed that macrophages (peritoneal and splenic) treated with anti-CCL2-neutralizing Ab displayed an increase in apoptosis (Figure 7B) and a decrease in cell viability (Figure 7C) as compared with vehicle-treated cells. Conversely, the addition of recombinant CCL2 restored apoptosis of $\mathrm{Nfat}^{+/-}$splenic macrophages (Figure 7D), while the same concentration of recombinant IL- 6 or TNF- $\alpha$ failed to do so (data not shown). Taken together, these data suggest that NFAT5 promotes macrophage survival by inducing CCL2 production in human and murine systems.
$R A$ peripheral monocytes show increased survival but decreased apoptotic death. Monocytes derived from progenitors in bone marrow are an important source of synovial-resident macrophages (33), and recent evidence suggests that self-renewal macrophages give rise to another origin of many tissue-resident macrophages (33). It is believed, however, that resident macrophages do not recirculate into the periphery. Since RA is a systemic disease, we wondered a) whether RA peripheral monocytes show increased survival but decreased apoptotic death, like RA-SF macrophages and b) whether they reflect RA-SF macrophages when cell survival and proliferation as well as NFAT5 and CCL2 expression are simultaneously assessed in peripheral monocytes and synovial macrophages of RA patients. We found that expression levels of both NFAT5 and CCL2 were significantly higher in matched RA-SF CD14 ${ }^{+}$cells than in peripheral $\mathrm{CD} 14^{+}$cells, which were collected at the same time (Figure 8A and Supplemental Figure 6B). Very interestingly, NFAT5 expression in matched synovial $\mathrm{CD}^{+}{ }^{+}$cells correlated well with that detected in simultaneously obtained peripheral monocytes (Figure 8B), thus suggesting that 
A

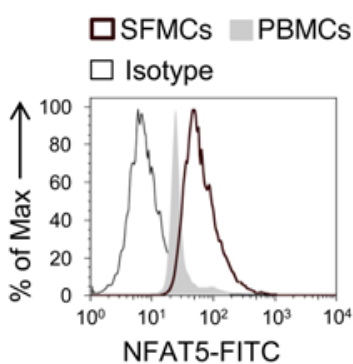

D

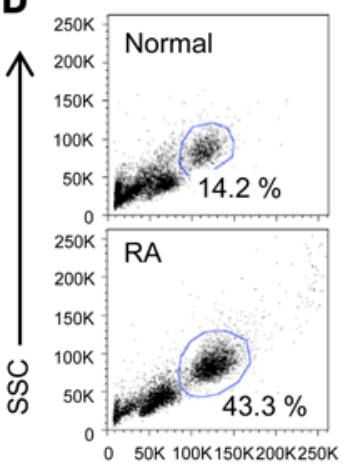

FSC
B

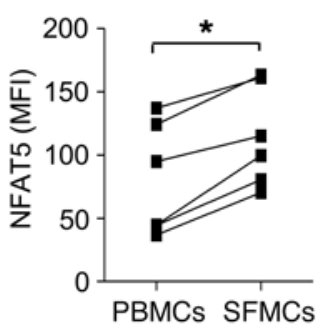

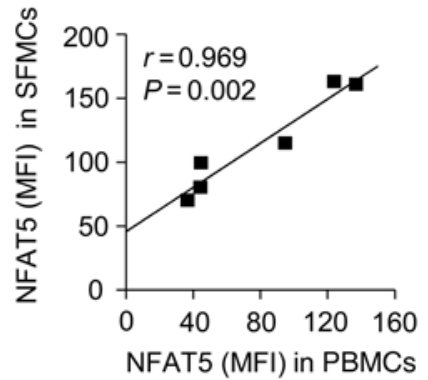

E

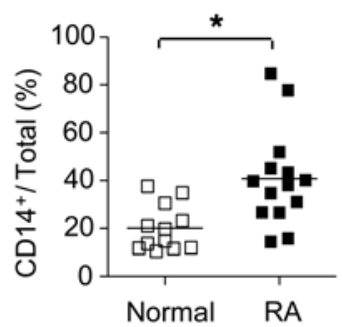

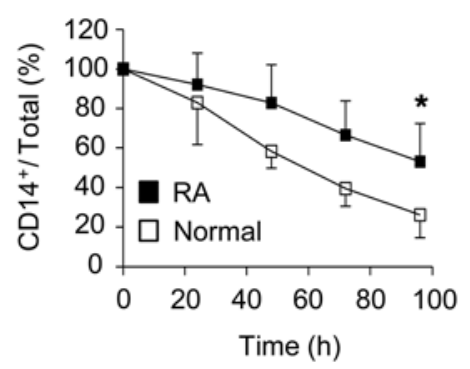

C

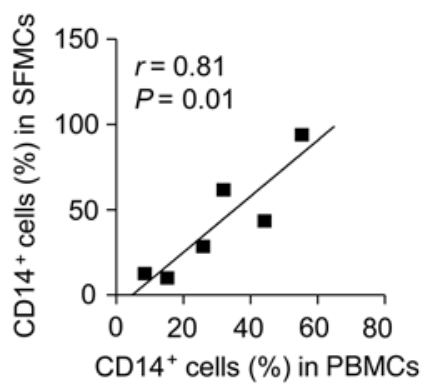

$\mathbf{F}$

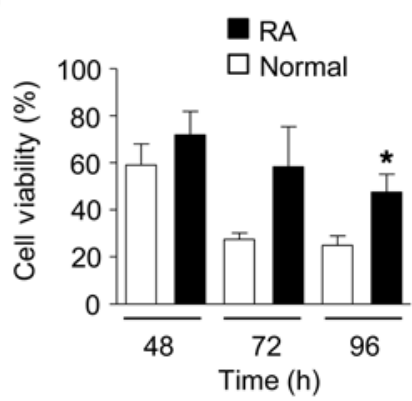

G

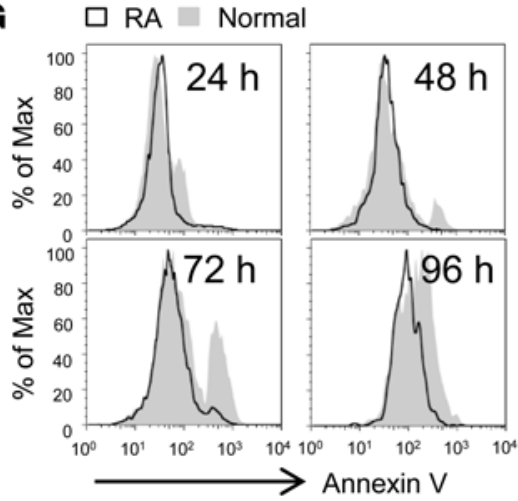

H

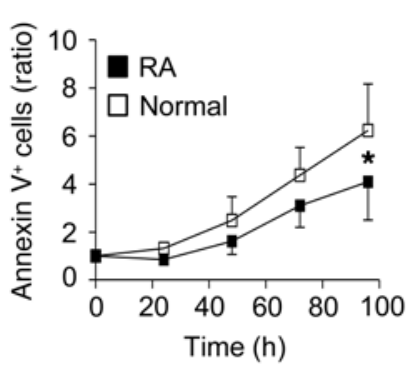

I

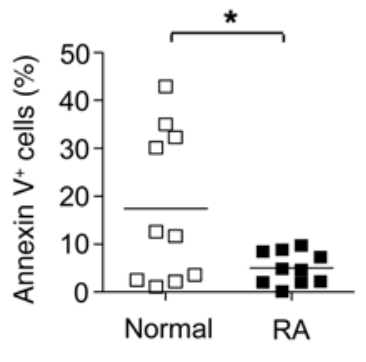

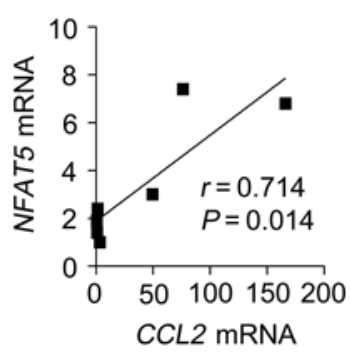

Figure 8. Peripheral CD14 ${ }^{+}$cells from RA patients are resistant to apoptotic death. (A and B) Comparison of NFAT5 expression levels in CD14 ${ }^{+}$cells between PBMCs and SFMCs from patients with RA $(n=6)$ (cells were obtained simultaneously). (C) Correlation of CD14 ${ }^{+}$cell frequencies between paired SFMCs and PBMCs from patients with RA, as assessed by flow cytometry. (D) Increase in the proportion of CD14+ cells in PBMCs from patients with RA. The percentages of CD14+ cells in PBMCs from RA patients $(n=14)$ and healthy controls $(n=12)$ were assessed by flow cytometry. (E) Time kinetics for the frequency of CD14 ${ }^{+}$ cells in PBMCs from RA patients $(n=5)$ versus normal controls $(n=4)$. PBMCs were cultured for 96 hours in RPMI 1640 containing $10 \%$ FBS. The proportion of CD14+ cells in PBMCs was determined by flow cytometry. (F) Increased viability of peripheral CD14+ ${ }^{+}$cells from patients with RA. CD14 ${ }^{+}$cells $\left(5 \times 10^{5}\right.$ cells), isolated from PBMCs from RA patients $(n=7)$ and healthy controls $(n=6)$, were cultured for 96 hours in RPMI 1640 containing $10 \%$ FBS. Cell viability was determined by MTT assay. (C) Time kinetics of apoptosis in CD14+ cells from RA patients $(n=6)$ versus healthy controls $(n=6)$. CD14 cells $\left(5 \times 10^{5}\right.$ cells) were cultured for 96 hours in RPMI 1640 containing 10\% FBS. Cell apoptosis was determined by flow cytometry for annexin V. (H) Decrease in the proportion of apoptotic CD14+ cells in PBMCs from patients with RA. Mononuclear cells were isolated from the peripheral blood of RA patients $(n=11)$ and healthy controls $(n=11)$. The proportion of annexin $\mathrm{V}^{+}$cells in $\mathrm{CD} 14^{+}$cells was assessed by flow cytometry immediately after isolating the PBMCs. (I) A positive correlation between NFAT5 and CCL2 mRNA levels was found in CD14+ cells from patients with RA. Data in $\mathbf{A}$ and $\mathbf{D}-\mathbf{H}$ represent the mean $\pm \mathrm{SD}$. (A and $\mathbf{D}-\mathbf{H}){ }^{*} P<0.05$, by Mann-Whitney $U$ test. (B, C, and I) Statistical significance was determined by Spearman's correlation analysis.

NFAT5 positivity in RA-SF macrophages, in part, comes from the peripheral NFAT5 ${ }^{\text {hi }}$ monocytes. In addition, the frequency of $\mathrm{CD}^{+} 4^{+}$cells in PBMCs correlated well with that of paired synovial fluid mononuclear cells (SFMCs) in patients with RA (Figure 8C), and the mean level of $\mathrm{CD} 14^{+}$cells was 2-fold higher in RA patients than in healthy controls (Figure 8D). In parallel, when cultured in RPMI 1640 media with 10\% FBS for 96 hours, the frequency of peripheral CD14 ${ }^{+}$cells was significantly higher in RA patients than in healthy controls (Figure 8E). Moreover, peripheral monocytes from patients with RA showed a lesser degree of apoptotic death than did those from healthy controls when cultured for 96 hours (Figure 8, F and G). Basal levels of apoptosis also were significantly lower in peripheral $\mathrm{CD} 14^{+}$monocytes from RA patients than in those from healthy controls (Figure 8H). Similar to the data 
A

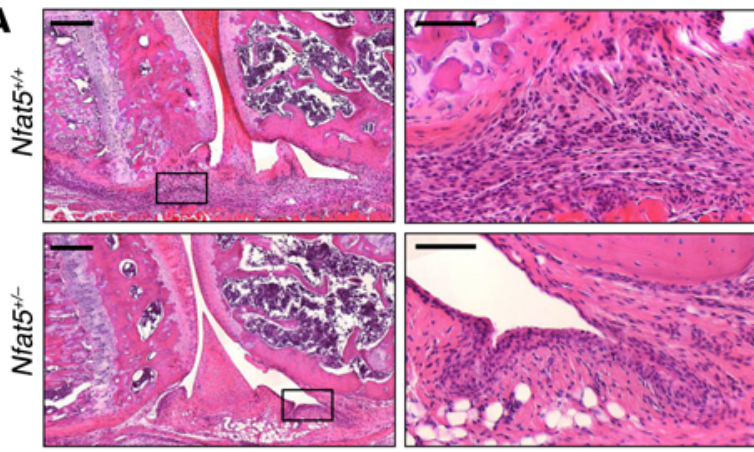

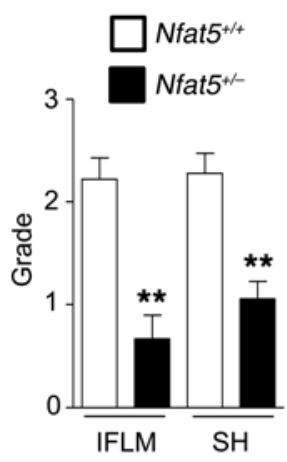

B

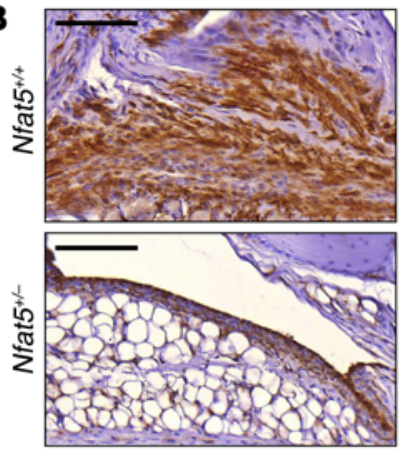

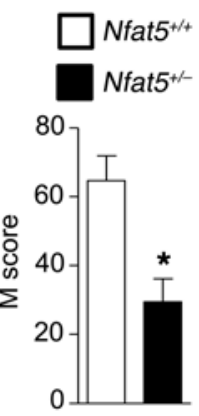

C
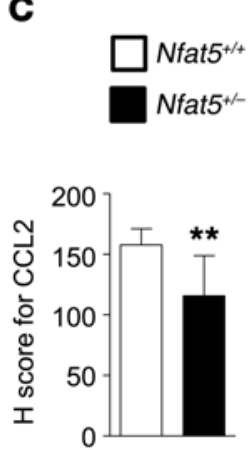

D
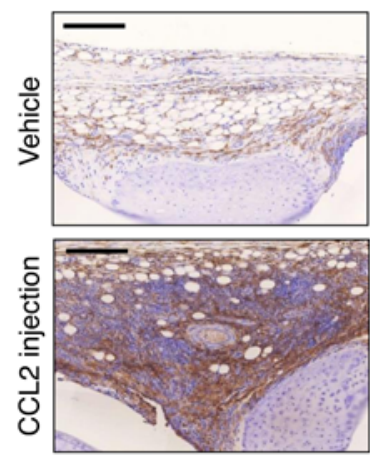

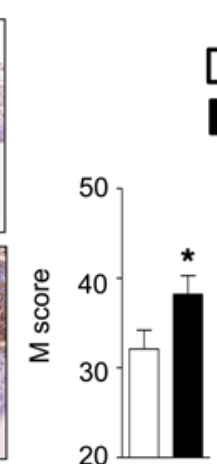

Vehicle

CCL2 injection

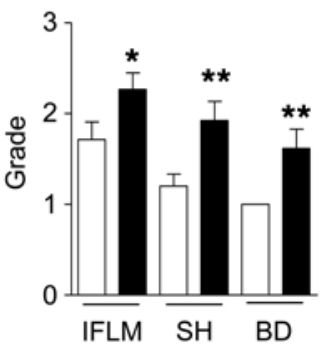

Figure 9. In vivo role of the NFAT5/CCL2 axis in macrophage infiltration and arthritis progression. (A) Effect of NFAT5 haploinsufficiency on arthritis severity in mice with mBSA/IL-1 $\beta$-induced arthritis. Joint sections from WT Nfat5+/+ and $\mathrm{Nfat}^{+/-}$mice were stained with $\mathrm{H} \& \mathrm{E}$, and arthritis severity was graded from 0 to 3 on day 7 (see the Supplemental Methods for additional details). The rectangular areas in the left panels are shown at higher magnification in the right panels. Scale bar: $200 \mu \mathrm{m}$ (left panels) and $100 \mu \mathrm{m}$ (right panels). Bar graph shows the mean \pm SD of the histological grade ( $n=5$ per group). ${ }^{* *} P<0.001$ between groups, by Mann-Whitney $U$ test. (B) Reduced infiltration of macrophages from Nfat5 ${ }^{+/-}$mice with mBSA/IL-1 3 -induced arthritis. Infiltrated macrophages were stained using a macrophage marker Ab (RM0029-11H3), and their numbers are expressed as the $M$ score, which is the product of staining frequency (percentage) multiplied by the intensity. Bar graph shows the mean \pm SD. Scale bars: $100 \mu \mathrm{m}$. ${ }^{*} P<0.01$, by Mann-Whitney $U$ test. (C) Reduced CCL2 expression in the knee joints of $N f a t 5^{+/-}$mice with mBSA/IL-1 $\beta$-induced arthritis. Expression levels were assessed by IHC using anti-CCL2 $\mathrm{Ab}$ (ab25124). The $\mathrm{H}$ score is the product of staining frequency (percentage) multiplied by the intensity and is presented as the mean $\pm \mathrm{SD}$ ( $n=5$ per group). ${ }^{* *} P<0.01$, by Mann-Whitney $U$ test. (D) Increase in arthritis severity by intra-articular injection of CCL2. Recombinant CCL2 ( $\left.2 \mu \mathrm{g}\right)$ was injected once daily on days 1,3 , and 5 into the knee joints of $\mathrm{Nfat}^{+/-}$mice. The joint sections were stained with $\mathrm{H} \& \mathrm{E}$ and macrophage marker Ab on day 7. Bar graphs show the mean $\pm \mathrm{SD}\left(n=5\right.$ per group). ${ }^{*} P<0.05$ and ${ }^{* *} P<0.01$, by Mann-Whitney $U$ test. Scale bars: $100 \mu \mathrm{m}$. BD, bone destruction; IFLM, inflammatory cell infiltration; $\mathrm{SH}$, synovial hyperplasia.

on synovial CD14+ cells (Figure 6D), NFAT5 mRNA expression in peripheral CD14 ${ }^{+}$cells correlated well CCL2 mRNA expression (Figure 8I). Collectively, these observations suggest that peripheral monocyte numbers are increased in patients with RA and show apoptotic resistance and increased survival, which might be associated with increased expression of NFAT5.

NFAT5 in macrophages is essential for the progression of chronic arthritis in mice. To ascertain the effect of the NFAT5/CCL2 axis in macrophages on chronic arthritis, we next generated methylated BSA/IL-1 $\beta$-induced (mBSA/IL-1 $\beta$-induced) arthritis, a model of chronic arthritis in which macrophages play a central role (34), in $\mathrm{Nat}^{+/-}$mice and their WT littermates. As shown in Figure 9, A and B, inflammatory cell infiltration, including macrophage infiltration and synovial hyperplasia, was significantly lower in NFAT5 haploinsufficient mice than in WT $\mathrm{Nfat}^{+/+}$mice, indicating that NFAT5 plays a key role in arthritis progression in this model. We next investigated whether the decrease in arthritis severity in
NFAT5-deficient mice is dependent on CCL2. As expected, CCL2 expression was significantly lower in the joints of $\mathrm{Nat5}^{+/-}$mice than in those of $\mathrm{Nat}^{+/+}$mice (Figure 9C). When recombinant CCL2 was injected repeatedly into one of the affected joints of $N \mathrm{Nat}^{+/}{ }^{+-}$mice with IL-1 $\beta$-induced arthritis, we found that macrophage infiltration was significantly increased as compared with that in vehicle-treated mice (Figure 9D, left and middle panels). Moreover, the extent of inflammation and joint destruction was more severe in mice injected with CCL2 (Figure 9D, right panel), which confirms the essential role of the NFAT5/CCL2 axis in macrophage infiltration and arthritis progression in vivo.

Finally, we tested the direct role of NFAT5-sufficient macrophages in promoting arthritis severity. When splenic macrophages from WT $\mathrm{Nat}^{+/+}$mice were injected into the knee joints of $\mathrm{Nfat}^{+/+}$mice just prior to induction of mBSA/IL-1 $\beta$-mediated arthritis, the histological severity assessed on day 7 , including inflammatory cell infiltration, bone destruction, and synovial 
A

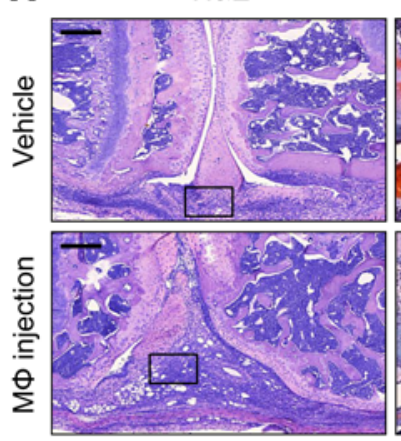

B

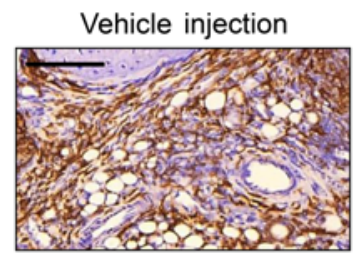
$M \Phi$ injection

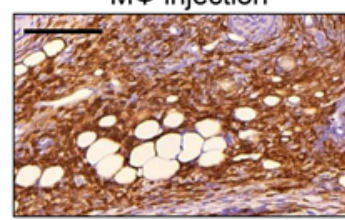

Safranin O

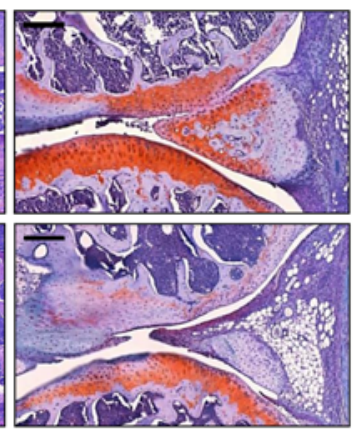

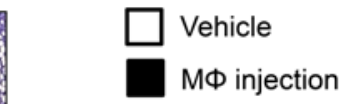

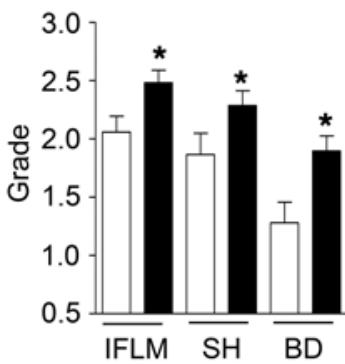

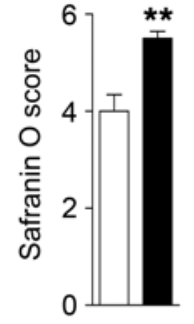

C
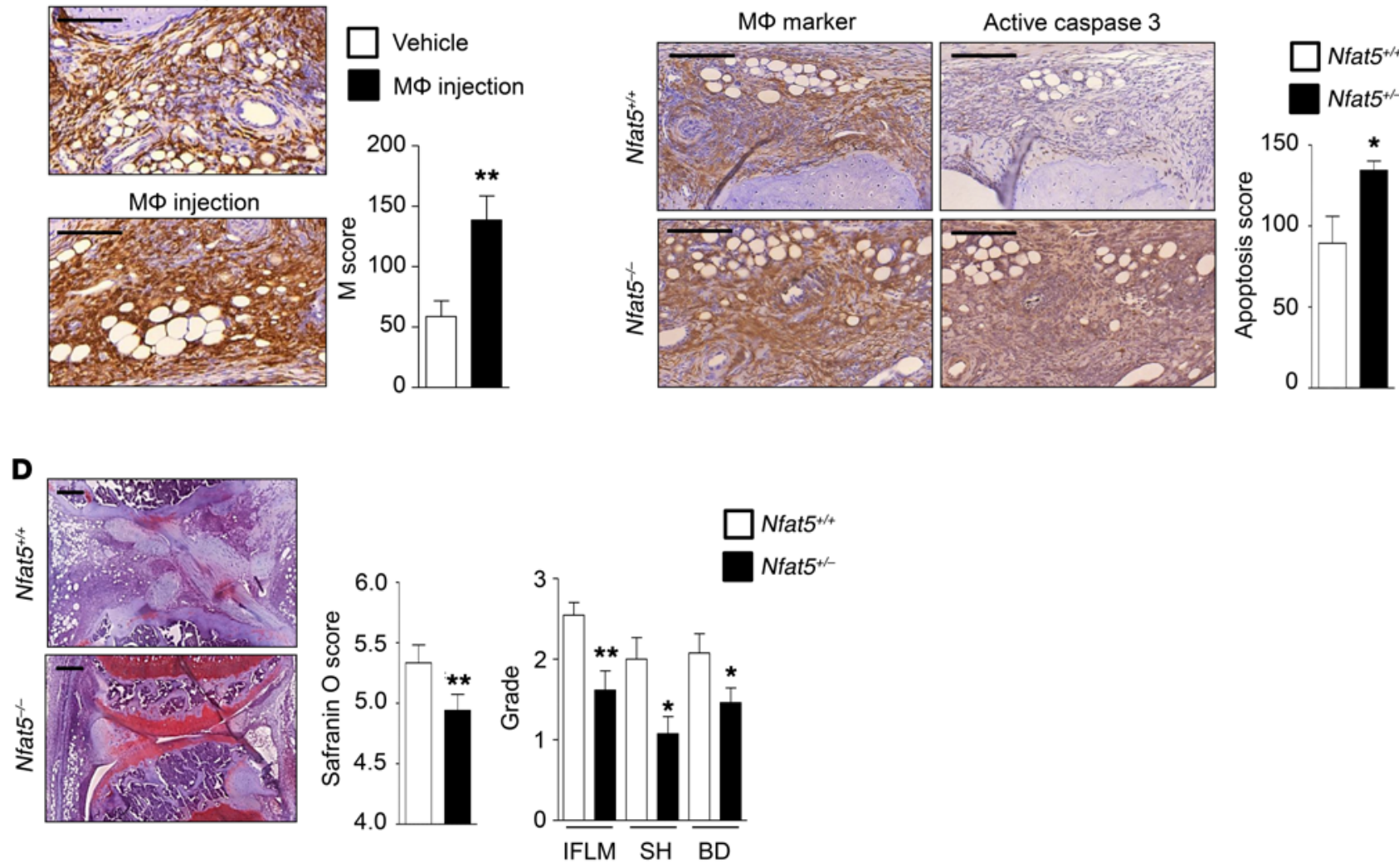

Figure 10. Direct in vivo effect of NFAT5 in macrophages on the progression of arthritis. (A and B) Increase in arthritis severity by intra-articular injection of NFAT5-sufficient macrophages. Activated macrophages $\left(5 \times 10^{5}\right.$ cells per mouse) stimulated with LPS (10 mg/kg) in vivo for 24 hours were isolated from the spleens of $\mathrm{Nfat}^{+/+}$mice and then injected intra-articularly into the knee joints of $\mathrm{Nfat5^{+/+ }}$ mice prior to injection of mBSA/IL-1 3 . The joint sections were stained with safranin 0 and macrophage marker $A b$ on day 7 to assess histological severity (A) and the degree of macrophage infiltration (B), respectively. Bar graphs show the mean \pm SD of the histological grades and $M$ scores ( $n=5$ per group). The rectangular areas in $\mathbf{A}$ are shown at higher magnification in B. ${ }^{*} P<0.01$ and ${ }^{* *} P<0.001$, by Mann-Whitney $U$ test. Scale bars: $200 \mu \mathrm{m}$ (A) and $100 \mu \mathrm{m}$ (B). (C and D) Increased apoptosis of macrophages in vivo due to NFAT5 deficiency. Activated splenic macrophages $\left(5 \times 10^{5}\right.$ cells) from $\mathrm{Nfat5}^{+/+}$and $\mathrm{Nfat}^{+/-}$mice were injected into the knee joints of $\mathrm{Nfat}^{+/-}$mice before the injection of $\mathrm{mBSA} / \mathrm{IL}-1 \beta$ ( $n=6$ per group). Arthritic joints were stained with macrophage marker Ab (left panel in C), anti-active caspase $3 \mathrm{Ab}$ (right panel in C), or safranin $O$ (D) on day 7. Active caspase 3 staining was performed on 4- $\mu \mathrm{m}$ tissue sections next to those stained with macrophage marker Ab from the same joint. Bar graphs show the mean \pm SD of histological grades assessed by safranin 0 staining (for the safranin 0 score, see the Supplemental Methods) and apoptosis scores. The apoptosis score was calculated as the staining frequency (percentage) with anti-active caspase 3 Ab multiplied by the intensity. ${ }^{*} P<0.05$ and ${ }^{*} P<0.005$, by Mann-Whitney $U$ test. Scale bars: $200 \mu \mathrm{m}$ (C) and $100 \mu \mathrm{m}$ (D).

hyperplasia, was significantly increased (Figure 10, A and B), demonstrating a direct effect of NFAT5-sufficient macrophages on the progression of chronic arthritis. To confirm that NFAT5 expression in macrophages is required for chronic arthritis, we injected $\mathrm{Nfat}^{+/-}$macrophages or WT $\mathrm{Nfat}^{+/+}$macrophages into the knee joints of $\mathrm{Nfat}^{+/-}$mice prior to the induction of mBSA/ IL-1 $\beta$-mediated arthritis and investigated the effect of these macrophages on arthritis severity. As shown in Figure 10C, on day 7, $\mathrm{Nfat}^{+/}$- macrophages showed more caspase 3 positivity than did WT $\mathrm{Nat}^{+/+}$macrophages. Moreover, inflammatory cell infiltra- 
tion, synovial hyperplasia, and bone destruction were less severe in mice injected with $\mathrm{Nfat5^{+/- }}$ macrophages than in mice with $\mathrm{Nat5}^{+/+}$macrophages (Figure 10D). These results indicate that NFAT5-deficient macrophages are more susceptible to apoptosis in vivo and are therefore less efficient at promoting joint destruction than are NFAT5-sufficient macrophages. Collectively, it can be suggested that NFAT5 in macrophages is necessary for the progression of chronic arthritis.

\section{Discussion}

Macrophages possess broad proinflammatory, destructive, and remodeling capabilities and actively participate in the perpetuation of chronic inflammation and joint destruction in RA $(7,8)$. In RA synovia, the number of synovial macrophages is positively correlated with disease activity $(10,11)$. Moreover, a change in synovial sublining macrophages is a sensitive biomarker for response to antirheumatic treatment in patients with RA (16). Also, elimination of macrophages by clodronate-containing liposomes induces long-term amelioration of rat adjuvant arthritis (35). A single intra-articular administration of clodronate liposomes decreases the expression of adhesion molecules in the synovial lining in RA patients, which correlates with macroscopic synovitis and C-reactive protein levels (21). Evidence is also emerging that the apoptotic death of activated macrophages is an important mechanism for controlling inflammation (36) and that dysregulated apoptosis can be one of the major pathogenic mechanisms responsible for RA pathology $(12,14)$. As such, the identification of key molecules that regulate the apoptosis and survival of macrophages may hold promise for the development of antimacrophage therapy for RA.

Through global gene expression profiling, we demonstrated that macrophage apoptosis, survival, and proliferation were the major pathologic processes represented by the DEGs in RA macrophages and NFAT5-deficient RAW 264.7 macrophages. Functional experiments in vitro showed that NFAT5 influenced the apoptotic death and proliferation of these cells. Interestingly, transcriptomic analysis of RA macrophages revealed that NFAT5 is a key TF in RA macrophage pathology and may have greater significance than $\mathrm{NF}-\kappa \mathrm{B}$ in RA pathogenesis. Indeed, NFAT5 expression was higher in RA macrophages than in normal macrophages differentiated from peripheral monocytes. Moreover, both NFAT5-deficient RA macrophages and mouse macrophages had higher rates of apoptotic death upon starvation or treatment with apoptosis-inducing CHX, SNP, and/or TG than did NFAT5-sufficient macrophages. Taken together, these results suggest that expression levels of NFAT5 and its target genes are increased in RA macrophages and play an essential role in the antiapoptosis and survival of macrophages in both human and murine systems. Given the importance of macrophage survival in RA progression (10-12, 14), NFAT5 inhibition, for example, via shRNA or small-molecule intervention, may retard RA progression by leaving macrophages more vulnerable to apoptosis.

To explore the possible mechanisms for increased NFAT5 expression in RA macrophages, we hypothesized that, since ambient proinflammatory and hypoxic stimuli are abundant in RA joints, they might also be contributing to increased levels of NFAT5 in RA macrophages. Our study demonstrated that NFAT5 could be activated by factors other than hypertonicity and that the proinflammatory and hypoxic stimuli, including M-CSF, IL-1 $\beta$, LPS, and $\mathrm{CoCl}_{2}$, represented a distinct modality for NFAT5 upregulation in macrophages. An emerging concept that defines the role of macrophages in RA is the notion of their high plasticity during development. M1 (classical, inflammatory) and M2 (alternative, antiinflammatory, tissue repair) represent two extremes of a spectrum of possible macrophage activation statuses (37). Here, M1-polarizing stimuli (IFN- $\gamma$ plus LPS), but not an M2-polarizing stimulus (IL-4), further increased NFAT5 expression in M-CSFdifferentiated $\mathrm{CD}_{1}{ }^{+}$cells. In the RA setting, it is thought that the most efficacious macrophage inhibition or depletion therapy will be the one that selectively targets proinflammatory M1 rather than antiinflammatory M2 macrophages (38). Our data define, for the first time to our knowledge, the selective association of M1, but not M2, polarization with NFAT5 induction and suggest that proinflammatory M1-polarizing stimuli and hypoxic conditions are the major contributing factors in the enhancement of NFAT5 expression in RA macrophages.

CCL2 is a classical inflammatory chemokine (39), and its expression is transcriptionally upregulated by PDGF, VEGF, and LPS (39). CCL2 is expressed by a variety of cell types, including leukocytes, smooth muscle cells, endothelial cells, fibroblasts, epithelial cells, and certain tumor cell lines (39). Recent studies have demonstrated that CCL2 promotes the survival of human CD11b ${ }^{+}$PBMCs (24) and prostate cancer PC3 cells (40). Synovial macrophages are the dominant source of CCL2 production (9), and CCL2 concentrations correlate with IL-1 $\beta$, IL-6, and IL-8 levels in RA-SF $(9,41)$.

Here, we demonstrated in the network model represented by the DEGs in RA-SF macrophages that CCL2 significantly interacted with the first neighbors that are associated with cell proliferation, survival, and antiapoptosis. As reported previously $(4,5)$, RAW 264.7 macrophages, primary mouse macrophages, and RA macrophages that were deficient in NFAT5 all showed a marked impairment in CCL2 production. In addition, CCL2 expression correlated well with NFAT5 expression in RA-SF macrophages and RA synovial tissues. Recombinant CCL2 almost completely restored apoptotic death of NFAT5-deficient macrophages in human and mouse systems; conversely, anti-CCL2-neutralizing Ab increased apoptosis of peritoneal and/or splenic macrophages in vitro. Moreover, when recombinant CCL2 was injected into one of the affected joints of $\mathrm{Nfat}^{+/-}$mice, joint destruction as well as macrophage infiltration were substantially increased. Taken together, our work is the first to our knowledge to demonstrate that the NFAT5/CCL2 axis is crucial to macrophage survival and suggests that CCL2 is secreted by RA macrophages in an NFAT5-dependent manner and contributes to RA pathology by conferring apoptotic resistance to synovial macrophages in an autocrine and paracrine manner. Notwithstanding, since NFAT5 regulates a number of genes involved in RA macrophage apoptosis in addition to CCL2, including CD74, $S O D 2, A P A F 1, D A P 3, S T K 3$, and FAS (Figure 6A), it is unlikely that CCL2 alone entirely mediates the NFAT5 promotion of RA macrophage survival. Multiple genes governed by NFAT5 may cooperatively contribute to this process.

NFAT5 has been implicated in the pathogenesis of autoimmune diseases, particularly in RA (22). We have uncovered a crucial role for NFAT5 in the development of autoimmune chronic arthritis (22). NFAT5 is highly expressed in the synovia of patients with RA and 
regulates synoviocyte proliferation and angiogenesis, a pathologic hallmark of RA (22). NFAT5-deficient mice show a marked reduction in anti-type II collagen Ab-induced arthritis (22). A study has also shown that partial loss of NFAT5 leads to lymphoid hypocellularity and impaired antigen-specific Ab responses (2). Most recently, it has been shown that high salt promotes the differentiation of $\mathrm{CD}^{+} \mathrm{T}$ cells into Th17 cells, important cells in the pathogenesis of autoimmune diseases, via the NFAT5 pathway and enhances the production of pathogenic Th17-related cytokines and chemokines (42). In the present study, we discovered what we believe to be a new role for NFAT5 in the progression of arthritis by showing that NFAT5 deficiency reduced macrophage infiltration and bone destruction in the mBSA/IL-1 $\beta$-induced arthritis model, a chronic arthritis model in which macrophages play a central role (34). Additionally, we found that NFAT5-deficient macrophages were more susceptible to apoptosis and less efficient at promoting joint destruction than were NFAT5-sufficient macrophages. Together, our work provides, to our knowledge, the first direct evidence in vivo that NFAT5 expression in macrophage enhances chronic arthritis by conferring apoptotic resistance to activated macrophages.

In summary, through global transcriptomic profiling of NFAT5-deficient RAW 264.7 macrophages and RA-SF macrophages, we have identified an NFAT5-centered molecular network that defines the antiapoptosis, survival, and proliferation of macrophages. Transcriptomic analysis revealed that NFAT5 is a key TF governing pathologic processes in RA macrophages and showed a more significant overlap between its regulon and DEGs than did TP53 or NF- $\mathrm{KB}$ complexes. Proinflammatory M1-polarizing stimuli and hypoxic conditions contribute to the enhanced NFAT5 expression seen in RA macrophages. Functional studies in vitro demonstrated that NFAT5 promotes survival and prevents apoptosis in both human and murine macrophages. CCL2, a chemokine, is critically involved in macrophage survival, acting as a representative downstream target of NFAT5. Moreover, NFAT5 expression in macrophages is necessary for the progression of chronic arthritis in mice. Our data suggest that NFAT5 regulates macrophage survival by inducing CCL2 secretion and thereby plays a central role in the pathogenesis of macrophage-dependent chronic inflammatory diseases such as RA. We anticipate that our systems approach to rheumatoid synovitis may serve as a comprehensive basis for further study of the fundamental mechanism(s) underlying activated macrophages in the pathogenesis of chronic inflammatory diseases.

\section{Methods}

Patients. One hundred seven patients who fulfilled the 1987 revised criteria of the American Rheumatism Association for RA (43) were studied. Clinical information was available for 104 (97.2\%) patients. The mean age of the 104 RA patients ( 13 men and 91 women) was $57.9 \pm 11.6$ years. The mean disease duration was $1.87 \pm 0.33$ years. A total of $85(81.7 \%)$ of 104 RA patients were positive for rheumatoid factor and 91 patients (87.5\%) were positive for the anticyclic citrullinated Ab. Eighty-two (78.8\%) patients had erosions on hand and foot $\mathrm{x}$-rays. The mean disease activity, as assessed by the disease activity score 28 (DAS28) (44), was $3.55 \pm 1.27$. All RA patients were treated with disease-modifying antirheumatic drugs (DMARDs) including methotrexate (77.9\%), antimalarial drugs $(50.0 \%)$, leflunomide $(50.0 \%)$, sulfasalazine $(13.5 \%)$, bucillamine (8.6\%), anti-TNF- $\alpha \mathrm{Ab}$ (6.7\%), and anti-IL-6 receptor $\mathrm{Ab}$ (tocilizumab, 8.6\%). Comparisons were made with 96 healthy controls (16 men and 80 women) who had no rheumatic diseases. The mean age of the healthy controls was $56.8 \pm 10.2$ years. There was no difference in age or sex between RA patients and healthy controls.

Generation of NFAT5-deficient cells. RAW 264.7 macrophages were obtained from the Korean Cell Line Bank (Seoul National University, Seoul, Republic of Korea). NFAT5-deficient RAW 264.7 macrophages were generated by stable transduction of $N f a t 5$ shRNA (NFAT KD) or by stable transfection of an NFAT5 decoy, as described previously (2) and as detailed in the Supplemental Methods.

Isolation of primary human and mouse macrophages. Monocytes/macrophages were obtained from PBMCs of healthy controls or RA patients using anti-CD14 magnetic beads (Miltenyi Biotec) according to the manufacturer's instructions. To induce differentiation into macrophages, monocytes from healthy controls were cultured with M-CSF $(20 \mathrm{ng} / \mathrm{ml})$ for 48 hours and then stimulated again for 24 hours with IL-4 $(20 \mathrm{ng} / \mathrm{ml})$ for M2 macrophages or with IFN- $\gamma(20 \mathrm{ng} / \mathrm{ml})$ plus LPS $(100 \mathrm{ng} / \mathrm{ml})$ for M1 macrophages. RA synovial macrophages were freshly isolated from SFMCs of RA patients using anti-CD14 magnetic beads. In some experiments, mouse peritoneal macrophages were isolated from the peritoneal fluid of WT $\mathrm{Nfat}^{+/+}$or $\mathrm{Nfat}^{+/-}$mice 3 days after inducing acute inflammation by injection of $3 \%$ sterile thioglycollate solution (BD Biosciences), as described previously (45). Primary mouse macrophages were also obtained from splenic cells 1 day after i.p. injection of LPS $(10 \mathrm{mg} / \mathrm{kg})$.

Microarray analysis. Total RNA was prepared using the RNeasy Mini Kit (QIAGEN), and RNA integrity was assessed using the Bioanalyzer 2100 (Agilent Technologies). The RNA integrity number (RIN) was near 10 for all samples (data not shown). RNA was then amplified and hybridized to the Illumina MouseWG-6 version 2.0 for mouse RAW 264.7 macrophages or to the Illumina Human Ref-8 version 3 Expression Bead Chip for human macrophages, according to the manufacturer's standard protocols. Probe intensities were normalized using the quantile normalization procedure (46). The microarray data were deposited in the NCBI's Gene Expression Omnibus (GEO) database (GEO GSE76554 and GSE49604).

Empirical hypothesis testing. A permutation test strategy was used to determine the significance of overlap between DEGs in RA-SF macrophages and NFAT5 target genes in RA-FLS and/or HUVECs. A total of 100,000 random permuted samples were used to compute the empirical $P$ values of the overlapping DEGs.

$q R T-P C R$. Total RNA was subjected to cDNA synthesis using the RNeasy Mini Kit (QIAGEN) according to the manufacturer's instructions. qRT-PCR was performed in a MyCycler (Bio-Rad) using SYBR Premix (Bio-Rad) according to the manufacturers' instructions. GAPDH was used as the internal control for PCR amplification. Transcript levels were calculated relative to those of the controls and are expressed as $-\Delta \Delta \mathrm{Ct}$. The gene-specific primers used are listed in Supplemental Table 1.

ELISA for CCL2. The level of CCL2 in the culture supernatant was measured with an ELISA kit (R\&D Systems) according to the manufacturer's recommendations. The detection threshold limit for CCL2 was $7.8 \mathrm{pg} / \mathrm{ml}$.

Cell proliferation assay. The cell proliferation rate was measured using a BrdU Proliferation Assay Kit (Roche Diagnostics) according to the manufacturer's instructions. Manual cell counts were also performed after trypan blue exclusion.

Determination of cell viability and apoptosis. Cell viability was determined by MTT assay (Sigma-Aldrich) as described previously 
(47). Apoptosis of macrophages was assessed by flow cytometry using an FITC-Annexin V Apoptosis Detection Kit (Santa Cruz Biotechnology Inc.) according to the manufacturer's instructions.

Flow cytometric analysis for NFAT5 and CCL2. Intracellular expression of NFAT5 or CCL2 in macrophages was detected by flow cytometry. In brief, cells were stained with $\mathrm{PE}$-conjugated $\mathrm{Ab}$ against anti-human $\mathrm{CD} 14 \mathrm{Ab}$ for 1 hour, fixed, and then made permeable with a commercially available permeabilization kit (eBioscience). For measurement of intracellular NFAT5 expression, the cells were incubated with rabbit anti-human NFAT5 Ab for 1 hour and then stained with an APC-conjugated secondary Ab for 30 minutes. Cells were stained with APC-conjugated anti-human CCL2 Ab for 1 hour to determine intracellular CCL2 expression.

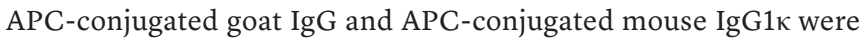
used as isotype-matched Abs. Fluorescence was measured using the FACS Canto II Flow System (BD Biosciences), and the data were analyzed using FlowJo software (Tree Star).

Induction of $\mathrm{mBSA} / \mathrm{IL}-1$-induced arthritis. Eight-week-old heterozygous $\mathrm{NFAT}^{+/-}$mice and their WT $\mathrm{NFAT}^{+/+}$littermates were used in the in vivo experiments. Following previously published protocols (34, 48), $10 \mu \mathrm{l}(20 \mathrm{mg} / \mathrm{ml}) \mathrm{mBSA}$ (Sigma-Aldrich) was singly injected into the knee joint on day 0 , followed by a daily s.c. injection of $20 \mu \mathrm{l}$ (250 ng) IL-1 $\beta$ (R\&D Systems) into the ipsilateral footpad on days 1 through 3.

Study approval. This study was performed with the approval of the IRB of Seoul St. Mary's Hospital (Seoul, Republic of Korea; CUMC09U034). All patients and healthy volunteers provided written informed consent to participate in the study.
Additional methods, including reagents and Abs, Western blot analysis for NFAT5, BCL-2, BAX, and cleaved caspase 8, immunofluorescence staining for NFAT5 and CCL2 expression, generation of BMDMs, histological assessment of arthritis, and statistical analyses are described in the Supplemental Methods.

\section{Author contributions}

SC, DK, SY, and SYC performed the experiments. WUK designed the experiments and analyzed the data. HMK provided the mice. HSK and CSC participated in sample collection. SC, DK, SY, DH, and CSC contributed to data analysis. SC and SY drafted the manuscript. WUK edited the manuscript. All authors commented on the manuscript. WUK coordinated the study design and implementation. YJP participated in sample collection and contributed to analysis of the data.

\section{Acknowledgments}

This study was supported by grants from the National Research Foundation of Korea (NRF), funded by the Ministry of Education, Science and Technology (NRF-2011-0020163, 2014R1A1A2055850, 2014R1A2A1A11049812, and 2015R1A3A2032927) and from the Institute for Basic Science (IBS-R013-G1), funded by the Korean Ministry of Science, Information and Communications Technology (ICT) and Future Planning.

Address correspondence to: Wan-Uk Kim, Department of Internal Medicine, Division of Rheumatology, The Catholic University of Korea, School of Medicine, Seoul, 137-701, Republic of Korea. E-mail:wan725@catholic.ac.kr.
1. Aramburu J, et al. Regulation of the hypertonic stress response and other cellular functions by the Rel-like transcription factor NFAT5. Biochem Pharmacol. 2006;72(11):1597-1604.

2. Kim NH, et al. The xanthine oxidase-NFAT5 pathway regulates macrophage activation and TLR-induced inflammatory arthritis. Eur JImmunol. 2014;44(9):2721-2736.

3. Halterman JA, Kwon HM, Wamhoff BR. Tonicity-independent regulation of the osmosensitive transcription factor TonEBP (NFAT5). Am J Physiol Cell Physiol. 2012;302(1):C1-C8.

4. Buxadé M, et al. Gene expression induced by Toll-like receptors in macrophages requires the transcription factor NFAT5. J Exp Med. 2012;209(2):379-393.

5 . Kim $\mathrm{NH}$, et al. Reactive oxygen species regulate context-dependent inhibition of NFAT5 target genes. Exp Mol Med. 2013;45:e32.

6. Iwamoto T, Okamoto H, Toyama Y, Momohara S. Molecular aspects of rheumatoid arthritis: chemokines in the joints of patients. FEBS J. 2008;275(18):4448-4455.

7. Burmester GR, Stuhlmüller B, Keyszer G, Kinne RW. Mononuclear phagocytes and rheumatoid synovitis. Mastermind or workhorse in arthritis? Arthritis Rheum. 1997;40(1):5-18.

8. Sack U, Stiehl P, Geiler G. Distribution of macrophages in rheumatoid synovial membrane and its association with basic activity. Rheumatol Int . 1994;13(5):181-186.

9. Koch AE, et al. Enhanced production of monocyte chemoattractant protein-1 in rheumatoid arthritis. JClin Invest. 1992;90(3):772-779.

10. Tak PP, et al. Analysis of the synovial cell infiltrate in early rheumatoid synovial tissue in relation to local disease activity. Arthritis Rheum. 1997;40(2):217-225.

11. Mulherin D, Fitzgerald O, Bresnihan B. Synovial tissue macrophage populations and articular damage in rheumatoid arthritis. Arthritis Rheum. 1996;39(1):115-124.

12. Liu H, Pope RM. The role of apoptosis in rheumatoid arthritis. Curr Opin Pharmacol. 2003;3(3):317-322.

13. Ma Y, et al. Fas ligation on macrophages enhances IL-1R1-Toll-like receptor 4 signaling and promotes chronic inflammation. Nat Immunol. 2004;5(4):380-387.

14. Pope RM. Apoptosis as a therapeutic tool in rheumatoid arthritis. Nat Rev Immunol. 2002;2(7):527-535.

15. Haringman JJ, Kraan MC, Smeets TJ, Zwinderman KH, Tak PP. Chemokine blockade and chronic inflammatory disease: proof of concept in patients with rheumatoid arthritis. Ann Rheum Dis. 2003;62(8):715-721.

16. Haringman JJ, et al. Synovial tissue macrophages: a sensitive biomarker for response to treatment in patients with rheumatoid arthritis. Ann Rheum Dis. 2005;64(6):834-838.

17. López-Rodríguez C, Aramburu J, Jin L, Rakeman AS, Michino M, Rao A. Bridging the NFAT and NF-kappaB families: NFAT5 dimerization regulates cytokine gene transcription in response to osmotic stress. Immunity. 2001;15(1):47-58.
18. Barabási AL, Gulbahce N, Loscalzo J. Network medicine: a network-based approach to human disease. Nat Rev Genet. 2011;12(1):56-68.

19. Lähdesmäki H, Shmulevich L, Dunmire V, Yli-Harja O, Zhang W. In silico microdissection of microarray data from heterogeneous cell populations. BMC Bioinformatics. 2005;6:54.

20. Li J, et al. Treatment of arthritis by macrophage depletion and immunomodulation: testing an apoptosis-mediated therapy in a humanized death receptor mouse model. Arthritis Rheum. 2012;64(4):1098-1109.

21. Barrera P, et al. Synovial macrophage depletion with clodronate-containing liposomes in rheumatoid arthritis. Arthritis Rheum. 2000;43(9):1951-1959.

22. Yoon HJ, et al. NF-AT5 is a critical regulator of inflammatory arthritis. Arthritis Rheum. 2011;63(7):1843-1852.

23. Trama J, Go WY, Ho SN. The osmoprotective function of the NFAT5 transcription factor in T cell development and activation. J Immunol. 2002;169(10):5477-5488

24. Roca H, Varsos ZS, Sud S, Craig MJ, Ying C, Pienta KJ. CCL2 and interleukin-6 promote survival of human $\mathrm{CD} 11 \mathrm{~b}+$ peripheral blood mononuclear cells and induce M2-type macrophage polarization. J Biol Chem. 2009;284(49):34342-34354.

25. Chung KY, Cheng IK, Ching AK, Chu JH, Lai PB, Wong N. Block of proliferation 1 (BOP1) plays an oncogenic role in hepatocellular carcinoma by promoting epithelial-to-mesenchymal transition. Hepatology. 2011;54(1):307-318. 
26. Wang Y, et al. Transcriptional repression of CDKN2D by PML/RAR $\alpha$ contributes to the altered proliferation and differentiation block of acute promyelocytic leukemia cells. Cell Death Dis. 2014;5:e1431.

27. Deng L, et al. Ran GTPase protein promotes human pancreatic cancer proliferation by deregulating the expression of Survivin and cell cycle proteins. Biochem Biophys Res Commun. 2013;440(2):322-329.

28. Starlets D, et al. Cell-surface CD74 initiates a signaling cascade leading to cell proliferation and survival. Blood. 2006;107(12):4807-4816.

29. Cavdar Koc E, et al. A new face on apoptosis: death-associated protein 3 and PDCD9 are mitochondrial ribosomal proteins. FEBS Lett. 2001;492(1-2):166-170.

30. Kawazoe N, Watabe M, Masuda Y, Nakajo S, Nakaya K. Tiam1 is involved in the regulation of bufalin-induced apoptosis in human leukemia cells. Oncogene. 1999;18(15):2413-2421.

31. Palmer EL, Miller AD, Freeman TC. Identification and characterisation of human apoptosis inducing proteins using cell-based transfection microarrays and expression analysis. $B M C$ Genomics. 2006;7:145.

32. Go WY, Liu X, Roti MA, Liu F, Ho SN. NFAT5/ TonEBP mutant mice define osmotic stress as a critical feature of the lymphoid microenvironment. Proc Natl Acad Sci USA. 2004;101(29):10673-10678.

33. Udalova IA, Mantovani A, Feldmann M. Macrophage heterogeneity in the context of rheumatoid arthritis. Nat Rev Rheumatol. 2016;12(8):472-485.

34. Yang YH, Hamilton JA. Dependence of interleukin-1-induced arthritis on granulocyte-macrophage colony-stimulating factor. Arthritis Rheum. 2001;44(1):111-119.

35. Kinne RW, et al. Long-term amelioration of rat adjuvant arthritis following systemic elimination of macrophages by clodronate-containing liposomes. Arthritis Rheum. 1995;38(12):1777-1790.

36. Tabas I. Macrophage death and defective inflammation resolution in atherosclerosis. Nat Rev Immunol. 2010;10(1):36-46.

37. Mantovani A, Sica A, Sozzani S, Allavena P, Vecchi A, Locati M. The chemokine system in diverse forms of macrophage activation and polarization. Trends Immunol. 2004;25(12):677-686.

38. Li J, Hsu HC, Mountz JD. Managing macrophages in rheumatoid arthritis by reform or removal. Curr Rheumatol Rep. 2012;14(5):445-454.

39. Kumar SN, Boss JM. Site A of the MCP-1 distal regulatory region functions as a transcriptional modulator through the transcription factor NF1. Mol Immunol. 2000;37(11):623-632.

40. Roca H, Varsos Z, Pienta KJ. CCL2 protects prostate cancer PC3 cells from autophagic death via phosphatidylinositol 3-kinase/AKT-dependent survivin up-regulation.J Biol Chem. 2008;283(36):25057-25073.

41. Harigai M, Hara M, Yoshimura T, Leonard EJ, Inoue K, Kashiwazaki S. Monocyte chemoattractant protein-1 (MCP-1) in inflammatory joint diseases and its involvement in the cytokine network of rheumatoid synovium. Clin Immunol Immunopathol.1993;69(1):83-91.

42. Kleinewietfeld M, et al. Sodium chloride drives autoimmune disease by the induction of pathogenic TH17 cells. Nature. 2013;496(7446):518-522.

43. Arnett FC, et al. The American Rheumatism Association 1987 revised criteria for the classification of rheumatoid arthritis. Arthritis Rheum. 1988;31(3):315-324.

44. Prevoo ML, van 't Hof MA, Kuper HH, van Leeuwen MA, van de Putte LB, van Riel PL. Modified disease activity scores that include twentyeight-joint counts. Development and validation in a prospective longitudinal study of patients with rheumatoid arthritis. Arthritis Rheum. 1995;38(1):44-48.

45. Zhang X, Goncalves R, Mosser DM. The isolation and characterization of murine macrophages. Curr Protoc Immunol. 2008; Chapter 14:Unit 14.1.

46. Bolstad BM, Irizarry RA, Astrand M, Speed TP. A comparison of normalization methods for high density oligonucleotide array data based on variance and bias. Bioinformatics. 2003;19(2):185-193.

47. Kong JS, et al. Inhibition of synovial hyperplasia, rheumatoid $\mathrm{T}$ cell activation, and experimental arthritis in mice by sulforaphane, a naturally occurring isothiocyanate. Arthritis Rheum. 2010;62(1):159-170.

48. Staite ND, Richard KA, Aspar DG, Franz KA, Galinet LA, Dunn CJ. Induction of an acute erosive monarticular arthritis in mice by interleukin-1 and methylated bovine serum albumin. Arthritis Rheum. 1990;33(2):253-260. 